\title{
Qualität in der Mittelohrchirurgie - eine kritische Standortbestimmung
}

\section{Quality in middle ear surgery - a critical position determination}

\section{다 (i) $($ ) $\odot$}

Autor

Marcus Neudert

Institut

Medizinische Fakultät Carl Gustav Carus, ERCD - Ear

Research Center Dresden an der Klinik und Poliklinik für

Hals-, Nasen- und Ohrenheilkunde, Kopf- und Hals-Chirurgie

Schlüsselwörter

Qualitätsbewertung, Ergebnisqualität, Tympanoplastik, Mittelohrrekonstruktion, Lebensqualität

Key words

Quality assessment, quality of the outcome, tympanoplasty, reconstruction of the middle ear, quality of life

\section{Bibliografie}

DOI https://doi.org/10.1055/a-1021-6427

Laryngo-Rhino-Otol 2020; 99: S222-S247

(c) Georg Thieme Verlag KG Stuttgart · New York

ISSN 0935-8943

Korrespondenzadresse

Prof. Dr. med. Marcus Neudert

Direktor: Prof. Dr. med. Dr. h. c. Thomas Zahnert

Univ. HNO-Klinik, Ear Research Center (ERCD)

Fetscherstr. 74

D-01307 Dresden

marcus.neudert@uniklinikum-dresden.de

\section{ZUSAMMENFASSUNG}

Zur Ergebnisbewertung der rekonstruktiven Mittelohrchirurgie nur die erzielte Verbesserung audiometrischer Messergebnisse heranzuziehen greift zu kurz. Auch wenn sie als funktionelle Parameter eine zentrale Stellung in der Therapiebewertung des Sinnesohrgans Ohr einnehmen, müssen sie für die moderne Qualitätsbeschreibung um eine Reihe von Faktoren ergänzt werden. Dabei sind die z. T. unterschiedlichen Perspektiven, aus denen ein qualitativ gutes Ergebnis bewertet wird, zu berücksichtigen. Aus Sicht des Patienten sind andere Faktoren ausschlaggebend als für den Arzt, für den Arzt wiederum andere als für die Kostenträger und die internationale otologische Gemeinschaft, die anhand von Ergebnissen gesicherte Erkennt- nisse aus mittelohrchirurgischen Studiendaten ziehen möchte, setzt wieder andere Kriterien an, um die Qualität zu bewerten. Dazu wird der allgemeine Qualitätsbegriff auf die Mittelohrchirurgie adaptiert. Zudem muss er auf unterschiedlichen Ebenen gedacht und die chirurgische Therapie von Mittelohrerkrankungen als ganzer Prozess verstanden werden. Damit fallen neue Aspekte in die Qualitätsbewertung, die eine strukturierte Beschreibung und Erfassung krankheitsspezifischer Symptome, Befunde und das Behandlungsergebnis umfassen. Auch die Nutzung international anerkannter Klassifizierungssysteme, um die eigenen Ergebnisse nicht nur publizierbar sondern auch Meta-Analyse-fähig zu machen muss in der heutigen Zeit als ein Qualitätsmerkmal angesehen werden. Hierzu stehen international erarbeitete und anerkannte Berichtssysteme zur Verfügung. Ihre Anwendung in der Routineversorgung macht die erhobenen Daten dabei nicht nur international vergleichbar, sondern ermöglicht auch die systematische Bewertung innerhalb einer Institution zur Qualitätsbeschreibung.

Neben audiologischen Messergebnissen werden chirurgische Qualitätsindikatoren betrachtet. Dabei wird der Blick auch auf eintretende Komplikationen und den Wert systematischer und strukturierter Erfassungssysteme gerichtet. Für die Nutzenbewertung durch die Patienten stehen mittlerweile validierte Messinstrumente zur Verfügung, deren Einsatz nicht mehr auf wissenschaftliche Studien beschränkt sein sollte. Zusammenfassend soll die Qualitätsbewertung nicht nur auf den „Patienten als Ganzes“, sondern auch den „Therapieprozess als Ganzes“, mit Einbeziehung von Merkmalen der Struktur- und Prozessqualität, ausgedehnt werden.

\section{ABSTRACT}

When evaluating the outcome of reconstructive middle ear surgery, it is insufficient to use only the achieved improvement of audiometric measurement results. Although, as functional parameters, they occupy a central position in the therapeutic assessment of the ear as a sensory organ, they must be supplemented by a number of modern quality control factors. Different perspectives for assessment of quality must be taken into account. What is important from the patient's point of view may not be the same factors as to the physician, while the phy- 
sician places a high value on factors that are less significant for the medical insurance company. The international otological community, who would like to draw conclusions from middle ear surgery data, might set different criteria altogether for assessing quality of surgery.

Hence, we propose to adapt the general concept of quality to middle ear surgery. This must be implemented on different levels and surgical therapy of middle ear diseases must be understood as a process. This means that new aspects must be included in quality assessment,

This means that quality assessment must comprise additional aspects, which include a structured description and recording of disease-specific symptoms, findings, and outcome of treatment. Furthermore, in today's world the use of internationally recognized classification systems must be regarded as a quality feature, in order to make results not only publishable but also capable of meta-analysis. Internationally developed and recognized reporting systems are available for this purpose. Their use in routine care not only makes the collected data internationally comparable, but also enables systematic evaluation within the institution for quality description and control.

In addition to audiological measurement results, surgical quality indicators are considered. We also focus on emerging complications and the value of systematic and structured evaluation and documentation systems. Validated measuring instruments are already available for patient benefit assessment, the use of which should no longer be limited to scientific studies. In summary, quality assessment of surgery should be extended to include not only the "patient as a whole", but also to the "therapy process as a whole", incorporating features of structural and process quality.

\section{Inhaltsverzeichnis}

$\begin{array}{ll} & \text { Zusammenfassung } \\ & \text { Abstract } \\ \text { 1. } & \text { Einleitung } \\ 2.1 & \text { Definition des Qualitätsbegriffs } \\ \text { 2.1.1 } & \text { Ergebnisqualität } \\ \text { 2.1.2 } & \text { Strukturqualität } \\ \text { 2.1.3 } & \text { Prozessqualität } \\ \text { 3. } & \text { Ergebnisqualität } \\ \text { 3.1 } & \text { Graft take-rate } \\ \text { 3.1.1 } & \text { Trommelfellrekonstruktion } \\ \text { 3.1.2 } & \text { Ossikuloplastik } \\ \text { 3.1.3 } & \text { Mastoidhöhlenobliterationen } \\ \text { 3.2 } & \text { Rezidivrate (Cholesteatomrezidive, -residuale) } \\ \text { 3.3 } & \text { Hörergebnisse } \\ \text { 3.3.1 } & \text { Reintonaudiometrie } \\ \text { 3.3.2 } & \text { Sprachaudiometrie } \\ \text { 3.3.3 } & \text { Erhebungszeitpunkte } \\ \text { 3.4 } & \text { Lebensqualität } \\ \text { 3.4.1 } & \text { Generische und spezifische Messinstrumente } \\ \text { 3.4.2 } & \text { HRQOL-Messinstrumente in der Mittelohrchirurgie }\end{array}$

3.4.3 Weitere Einflussfaktoren auf die HRQOL

3.4.4 Empfehlungen zu Auswahl und Einsatz von HRQOL-Messinstrumenten

3.5 Ergebnisqualität als Abwesenheit von Komplikationen (ein Paradigmenwechsel)

3.5.1 Zur Definition der Begrifflichkeiten „Fehler“ und „Komplikation“

3.5.2 Spezifische Komplikationen nach Ohroperationen

3.5.3 Retrospektive Komplikationsbetrachtung und prospektive Komplikationserfassung

4. Prozess- und Strukturqualität

4.1 Dokumentationsqualität

4.1.1 Unterschiede in Krankenversorgung und Forschung S238

4.1.2 Beschreibungs- und Dokumentationsstandards $\quad$ S238

4.1.3 Anwendung von Klassifikations-Systemen und Berichtsstandards

4.2 Erfassungs- und Dokumentationssysteme

4.2.1 Common Otology Audit Database

4.2.2 Standardized Korean Ear Surgery Database S241

4.2.3 Oto-Database S241

4.2.4 Otology-Neurotology Database $\quad$ S241

4.2.5 OtoKir Database $\quad$ S241

4.2.6 Swedish National Quality Registry for Myringoplasty S242

$\begin{array}{lll}4.2 .7 & \text { ENTstatistics } & \mathrm{S} 242\end{array}$

Literatur $\$ 243$

\section{Einleitung}

Der Qualitätsbegriff erfährt im Rahmen des vorliegenden Referatebandes eine vielfältige Beleuchtung. Dabei wird deutlich, dass er in unterschiedlichen Bereichen der Medizin teils sehr individuelle Definitionen aufweist. Bei der Therapie eines Sinnesorganes ist die Qualität der Behandlung in erster Linie am Funktionserhalt oder der -wiedererlangung ablesbar. Einige Qualitätsindikatoren scheinen daher auf der Hand zu liegen. Ist das Ziel eines operativen Eingriffs die Hörverbesserung, so sind selbstredend audiologische Ergebnisse im vorher- nachher Vergleich aussagekräftig und ermöglichen eine „objektive“ Messung (mit den Einschränkungen psychophysischer Messverfahren) des Operationserfolgs und mit- 
telbar seiner Qualität. Erweitert man das Spektrum an erfassten und möglichen Parametern stellt sich schnell die Frage nach der Sinnhaftigkeit - ist „mehr“ wirklich ein „mehr“ an Aussagekraft? Und wenn ja, welches ist eine geeignete Parameterauswahl um die Qualität einer Therapie in der Mittelohrchirurgie ausreichend zu beschreiben?

Andere Qualitätsindikatoren wiederum haben erst in den letzten Jahren überhaupt Einzug in die Ergebnisbewertung gefunden, da sie schwerer messbar und in einer durch Evidenz und Objektivität getriebenen akademischen Landschaft schwerer etablierbar sind. Die Messung der gesundheitsbezogenen, krankheitsspezifischen Lebensqualität gibt uns Ärzten aber die Möglichkeit, die Qualität zu messen, die der Patienten subjektiv empfindet. Dies kann unter Umständen von der Qualitätsbewertung des Therapeuten abweichen. Dieser Perspektivenwechsel lässt sich fortsetzen, wenn man nicht nur die Behandlungsqualität eines einzelnen Patienten bewerten möchte, sondern die einer Patientenkohorte. Hierfür sind schnell geeignete Instrumente und Verfahren nötig, um die rasch wachsenden Datenmengen zu verarbeiten und analysieren zu können. Insbesondere der in den letzten Jahren lauter gewordene Ruf nach prospektiven Studien stellt deutlich höhere Anforderungen an die Dokumentationsqualität in der Behandlung. Sollen die in der täglichen Behandlungsroutine gewonnen Daten direkt für wissenschaftliche Zwecke genutzt und ausgewertet werden, dann bedarf es standardisierter Erhebungs- und Dokumentationsinstrumente. Diese zu etablieren und zu pflegen stellt oft einen zusätzlichen Aufwand dar, der zeitlich und/oder monetär nicht vorgesehen ist. Nichtsdestotrotz bedarf es dieser Methoden, um auf dem Weg von der empirischen Medizin zu einer wissenschaftlich begründeten und fundierten Therapie zu kommen.

Im Folgenden soll versucht werden, etablierte und neue Qualitätsindikatoren, die für die Beschreibung der Behandlungsgüte in der Mittelohrchirurgie unmittelbar und mittelbar geeignet sind, geordnet zusammenzutragen. Dabei soll der Blick auch darauf gerichtet werden, wie die gewonnen Primärdaten verarbeitet und ausgewertet werden. Gerade in einer Zeit der „postfaktischen Politik“ scheint das Bekenntnis zu einer ehrlichen und detaillierten Ergebnisgewinnung, -verarbeitung und -beschreibung wichtiger denn je - denn auch darin spiegelt sich die Qualität der otologischen Gemeinschaft wider.

\section{Definition des Qualitätsbegriffs}

Der Qualitätsbegriff hat seit den 2000er Jahren eine immer größere Präsenz und Bedeutung in der Medizin bekommen. Mittlerweile beschäftigen sich ganze Abteilungen mit Qualitätsmanagement und Qualitätsmanagementbeauftragte (QMB) sind mit der Erstellung und Pflege von Qualitätshandbüchern, Prozessbeschreibungen und Audits beschäftigt. Ohne an dieser Stelle die Sinnhaftigkeit einer ohnehin nicht mehr rückgängig machbaren Entwicklung zu erörtern, soll doch die konkrete Frage nach den Implikationen für die Mittelohr-Chirurgie gestellt werden. Denn die Verwendung der Begriffe „-management“ und „-sicherung“ bedeutet, dass der Gegenstand dessen, was es zu managen und sichern gilt, klar definiert ist. Es muss Kenntnis über die Art der zu erhebenden Daten bestehen, unter welchen Umständen sie erhoben wurden und welche Unsicherheiten bei ihrer Messung, Dokumentation und Aus- wertung bestehen. Weiterhin müssen die Daten im Gesamtkontext der Bewertung eingeordnet und sinnvoll gewichtet werden [1].

Für eine systematische Bearbeitung des Themas soll die von Donabedian vorgeschlagene Unterteilung in Struktur-, Prozess- und Ergebnisqualität herangezogen werden, die sich als praktikabel erwiesen hat [2-4]. Das Spektrum an bereits etablierten und möglichen Qualitätsindikatoren, die in der Mittelohrchirurgie identifiziert werden können, lassen sich größtenteils in die letztgenannte Kategorie - die Ergebnisqualität - einordnen. Dies ist nachvollziehbar, denn am Ende zählt das für den Patienten erzielte Behandlungsergebnis. Die Evaluation der Struktur- und Prozessqualität stellt sich demgegenüber schon als deutlich schwerer heraus, da sie von den lokalen strukturellen Gegebenheiten und den individuellen Prozessen abhängen, unter denen die Therapie und damit auch die Ergebnisse produziert werden.

Dennoch gibt es in diesem Bereich Wege und Hilfsmittel die existierenden Struktur- und Prozessvariablen wenigstens hinreichend zu beschreiben, wenn schon nicht zu minimieren bzw. zu eliminieren. Dies zu tun ist der Bereich der kontrollierten Studien, die versuchen eine möglichst genau definierte Frage unter Ausschluss aller unkontrollierten Einflüsse zu untersuchen [5]. Die Krankenversorgung stellt aber eine klinische Routine mit dem Anspruch, höchste Behandlungsqualität zu liefern dar und unterliegt keinem speziell entworfenen Design einer randomisierten kontrollierten Studie. Dafür ist sie an Leit- und Richtlinien und an den ethischen und moralischen Grundzügen ärztlichen Handelns ausgerichtet.

\subsection{Kategorien des Qualitätsbegriffs in der Mittel- ohrchirurgie}

Welche Qualitätsindikatoren lassen sich unter den genannten Gesichtspunkten in der Mittelohrchirurgie identifizieren?

\subsubsection{Ergebnisqualität}

Unter dem Begriff der Ergebnisqualität werden alle Qualitätsindikatoren zusammengefasst, die sich am Resultat der Intervention orientieren und dieses beschreiben bzw. messbar machen. Hierzu zählen die klassischen funktionellen Parameter der Audiometrie, aber auch die unterschiedlichen Ausprägungen der „graft take-rate“ (GTR), also der Anteil an erfolgreich im Körper integrierter Trans- und Implantate. Eine in den letzten Jahren immer wichtiger Kategorie in der Ergebnisbewertung ist die gesundheitsbezogene Lebensqualität (HRQOL), die wissenschaftlich fundiert die subjektiv vom Patienten empfundene krankheitsspezifische Beeinträchtigung abbildet. Wird der Qualitätsbegriff „rückwärts“ gedacht, so kann die Abwesenheit von Komplikationen ebenfalls als Qualitätsindikator verstanden werden. Im übertragenen Sinn stellen die spezifischen Komplikationsraten reziproke Parameter der Ergebnisqualität dar. Daher werden in diesem Abschnitt zusätzlich allgemein anerkannte Komplikationen von Mittelohreingriffen beschrieben und hinsichtlich der in der Literatur verfügbaren Auftretenswahrscheinlichkeit untersucht.

\subsubsection{Strukturqualität}

Die Strukturqualität subsumiert die Beschreibung der Rahmenbedingungen, die Charakteristika der personellen und materiellen Ressourcen, die bei der Behandlung (Leistungserbringung) zur Verfügung stehen. Zum anderen sind aber auch organisatorische As- 
pekte, wie die zur Verfügung stehenden Arbeitskonzepte gemeint. Daher können hier die Vorhaltung und Nutzung von Dokumentationssystemen beschrieben werden, die zur standardisierten Beschreibung und effektiven Aus- und Bewertung von Patientendaten nutzbar sind. Diese nehmen in der Mittelohrchirurgie einen immer wichtigeren Platz ein, da sie klare Definitionen und Kategorien beinhalten, die eine übergeordnete Auswertung von Therapiedaten ermöglichen.

Die Strukturqualität beinhaltet aber auch Kenntnisse, Fähigkeiten, Kompetenzen und Qualifikationen, sowie den Aus-, Weiter-, und Fortbildungsstand des Personals. Hier können chirurgische Trainingsmodelle und -programme, die die Fertigkeiten der Operateure verbessern, strukturierte Fortbildungen und Kursangebote angeführt werden. Messbare Größen, die einen Qualitätsindikator darstellen können, existieren hierzu nur wenige. Zudem ist das Feld zu umfangreich, um im Rahmen dieser Arbeit behandelt werden zu können.

\subsubsection{Prozessqualität}

Die Prozessqualität umfasst alle ärztlichen und administrativen Tätigkeiten, die direkt oder indirekt am unmittelbaren Versorgungsprozess beteiligt sind. Hier können für die Mittelohrchirurgie die Handhabung und Umsetzung von etablierten Standards, Klassifikationen und guter wissenschaftlicher Praxis eingeordnet werden. Dieser Punkt ist eng mit den genannten Aspekten der Strukturqualität verknüpft und kann, gemeinsam mit diesem als Dokumentationsqualität zusammengefasst werden. Dieser nicht in den Qualitätsdimensionen von Donabedian definierte Begriff, umfasst die die Güte, mit der die Indikatoren der Ergebnisqualität in der Literatur beschrieben werden. Die Dokumentationsqualität hat aber einen direkten Einfluss auf die Aussagekraft der beschriebenen Ergebnisse, sodass sie eine entscheidende Grundlage der Ergebnisqualität darstellt.

\section{Ergebnisqualität}

Das Messen von Qualität am Ergebnis oder Outcome einer Maßnahme ist nachvollziehbar und effektiv. Im Rahmen der Mittelohrchirurgie lassen sich mehrere Erfolgsparameter definieren, an denen die Qualität der Versorgung messbar ist.

\subsection{Graft take-rate}

Der Anteil an Patienten oder Operationen, bei denen ein eingebrachtes Transplantat oder Implantat im Körper verbleibt und dort integriert bzw. nicht abgestoßen wird, wird als graft take-rate (GTR) bezeichnet. Dies lässt sich in der Mittelohrchirurgie auf den Erfolg einer Trommelfellrekonstruktion, einer eingebrachten Ossikuloplastik und den Verbleib von Obliterationsmaterial in Mastoidhöhlen beziehen. Bei jedem Aspekt finden sich primäre Ziele, die es zu erreichen gilt, wie bspw. einen stabilen und dauerhaften Trommelfellverschluss, wenn eine Trommelfellrekonstruktion durchgeführt wurde. Geeignete Messgrößen um den Erfolg zu messen sind in diesem Fall der Anteil an Rezidivperforationen, Retraktionen oder, in begrenztem Maß, die postoperative Schallleitungsschwerhörigkeit (Air-Bone-Gap (ABG)). Im Folgenden sollen daher die einzelnen, für eine qualitativ hochwertige Mittelohrchirurgie zu berücksichtigenden Faktoren genauer beleuchtet werden.

\subsubsection{Trommelfellrekonstruktion}

Ziele: $\quad$ stabiler, dauerhafter Paukenhöhlenverschluss, maximale Schallabsorption

Messgrößen: GTR, ABG, (Schwingungsfähigkeit)

Ziele der Trommelfellrekonstruktion sind der dauerhafte Abschluss der Paukenhöhle, um das physiologische Mittelohr-Kompartiment wiederherzustellen und dabei eine maximale Schallabsorption bei gleichzeitig höchstmöglicher Stabilität zu erreichen. GTR und Re-Perforationsrate verhalten sich gegenläufig.

Eine aktuelle Meta-Analyse (214 Studien, 26097 Patienten) weist eine 12 -Monats GTR von $86,6 \%$ unabhängig von Alter, Perforationsgröße und Rekonstruktionsmaterial auf [3]. Die Analyse einzelner Faktoren zeigt eine 5,8\% höhere Versagensrate bei Kindern. Zudem haben kleinere Perforationen ( $<50 \%$ der Trommelfellfläche) eine 6,1\% bessere Prognose, wie auch sich Knorpel als Rekonstruktionsmaterial gegenüber Faszie mit einer 2,8\% höheren Verschlussrate überlegen zeigt. Diesen Unterschied für Knorpel und Faszie belegt eine weitere Metanalyse (11 prospektive und 26 retrospektive Studien, 3606 Patienten), bei der eine GTR von $92 \%$ mit Knorpel und $82 \%$ mit Faszie erzielt $(p<0,001)$ wurde. Unterschiede im Anteil eines postoperativen $A B G<10 \mathrm{~dB}$ waren insgesamt zwischen den Gruppen nicht zu verzeichnen. Die isolierte Betrachtung der prospektiven Studien zeigte jedoch einen signifikanten Vorteil der Faszien-Rekonstruktion ( $p=0,02)$. Gegenläufig verhält sich dies bei Betrachtung der GTR, bei der die Knorpel-Rekonstruktion signifikant besser abschnitt $(p=0,001)[6]$.

Eine Otorrhoe zum Zeitpunkt scheint nur in der kurzfristigen Analyse ( 2 bis 6 Monate) einen nachteiligen Effekt zu haben. Hier zeigen sich 94,4\% der trockenen Ohren verschlossen gegenüber $84,8 \%$ der aktiv entzündeten ( $p=0,002)$. Im Langzeitintervall (>12 Monate) sind keine Unterschiede mehr hinsichtlich der GTR feststellbar $[3,7,8]$.

Eine vorzeitige Erhebung ( $<12$ Monate) der GTR führt zu falsch hohen Verschlussraten [8]. Eine prospektive Analyse von 837 operierten Ohren in einem einzelnen Zentrum zeigte 2 bis 6 Monate nach Operation eine GTR von 93,0\%, die auf 86,6\% nach 12 Monaten absank $(p<0,001)$. Dieser Effekt war auch nach Adjustierung für alle untersuchten prognostischen Faktoren mit einer mittleren Abnahme von 6,0\% nachweisbar. Ein Erhebungsintervall von mindestens 12 Monaten ist für eine verlässliche Angabe der GTR und einen Vergleich mit der internationalen Literatur nötig.

Angesichts der großen Datengrundlage, auf der die o.g. Ergebnisse basieren, können diese mit hoher Wahrscheinlichkeit als gesichert angesehen werden. Die beiden Instrumente Meta-Analyse und Datenbanksystem sind für die Generierung dieser Ergebnisse erforderlich.

Eine Beurteilung der Schwingungsfähigkeit ist derzeit mit den Mitteln der etablierten Diagnostik noch nicht möglich. Ein Ansatz, die postoperative Schwingungsfähigkeit des (rekonstruierten) Trommelfells zu messen stellt die optische Kohärenz Tomografie (OCT) dar [8-10].

Neben der prinzipiellen Zugänglichkeit und Beurteilbarkeit des Trommelfells mit der OCT, konnte in einem Patienten eine Schwingungsanalyse des Trommelfells mitsamt dem sichtbaren Prothesenteller durchgeführt werden ( $\mathbf{A}$ Abb. 1). Dabei zeigte die Abnahme der Schwingungsamplitude des Prothesentellers der gemessenen 
Schalleitungsschwerhörigkeit im Reintonaudiogramm (Morgenstern et al., 2019 (accepted). Auch wenn es sich hierbei noch um Einzelfallstudien mit hohem Bearbeitungsaufwand handelt, könnte dieses Verfahren in Zukunft die Mittelohrdiagnostik um die detaillierte Schwingungsanalyse in vivo bereichern.

\subsubsection{Ossikuloplastik}

Ziele: $\quad$ gute und dauerhafte Schallübertragung

Messgrößen: ABG, Prothesen-Extrusions-Rate, (Schwingungsfähigkeit)

Die Ergebnisse nach Rekonstruktion des Schalleitungsapparats werden von vielen Faktoren beeinflusst. Dabei sind die Themen der Biomechanik des Mittelohres [12-15], die Materialfrage der Prothesen [16-18] und der Operations- sowie Rekonstruktionstechniken $[13,15,16,19,20]$ bereits ausführlich beleuchtet. Im klinischen Diskurs lässt sich die Frage nach einer erfolgreichen Ossikuloplastik strenggenommen auf die beiden Indikatoren des postoperativen ABG und der Versagensrate, in diesem Fall die Extrusionsrate von Mittelohrprothesen, reduzieren. Obgleich einzelne Rekonstruktionstechniken und -materialien in experimentellen Untersuchungen Vorteile im Übertragungsverhalten bieten mögen, führen die Störeinflüsse im pathologisch veränderten oft zu einer Abschwächung derartiger Unterschiede [21].

Auch hier bietet das Instrument der Metaanalyse die Möglichkeit, Effekte aus mehreren geeigneten Studien zusammenzuführen und gebündelt zu betrachten. Die Frage nach qualitativen Unterschieden zwischen Partial- (PORP) und Totalprothesen (TORP) bezogen auf den postoperativen ABG und die Extrusionsrate untersuchte 2013 eine Meta-Analyse von 40 Studien (4311 Patienten; 2344 PORP und 1967 TORP) [22]. Dabei zeigten die PORP einen durchweg geringeren ABG ( $<20 \mathrm{~dB}$ ) als die TORP, auch nach Unterscheidung nach Operationstechnik, Prothesenmaterial und Beobachtungsintervall. Die Autoren unterstreichen demzufolge die Bedeutung der Stapessuprastruktur für eine stabile Rekonstruktion. Die Extrusionsrate verhielt sich in hier analog: PORP waren signifikant weniger von Prothesenextrusionen betroffen und den verglichen TORP überlegen.
Ein konkreter Vergleich von Titan-Prothesen und Nicht-Titan-Prothesen erbrachte in einer weiteren Meta-Analyse von 12 Studien (1388 Patienten; 621 Titan-Prothesen und 767 Nicht-Titan-Prothesen) keinen Unterschied gemessen am postoperativen ABG und der Extrusionsrate [23]. Bei der Untersuchung wurde ein verbleibender $A B G<20 \mathrm{~dB}$ als erfolgreiche Ossikuloplastik gewertet. Eine Aufteilung in PORP und TORP erbrachte ebenfalls keine Unterschiede im Hörergebnis zwischen den Gruppen. Gleiches gilt für die Betrachtung der Prothesen-Extrusionen, bei denen ebenfalls keine Unterschiede in den Gruppen- bzw. Subgruppen-Analysen auffielen.

Das zusätzliche Überfüttern der Prothesenkopfplatte mit Knorpel reduziert in mehreren Studien die Exstrusionsrate von TitanProthese [23-29] und kann daher als Standard angesehen werden.

Die Autoren beider Analysen diskutieren die Limitationen ihrer Untersuchungen offen. Allerdings sind diese nicht in der Methodik der Meta-Analyse, sondern in den Quelldaten, also den zur Analyse herangezogenen Primär-Studien zu suchen. Das Fehlen von generell prospektiven Studien, wie auch eine hinreichende Beschreibung der Studienpopulationen sind hier zu nennen. Zudem ist bemerkenswert, dass eine Vielzahl von Studien nicht berücksichtigt werden konnte, weil die Daten in ihrer Darstellung nicht Meta-Analyse-fähig waren.

\subsubsection{Mastoidhöhlenobliterationen}

Ziele: $\quad$ kleines Höhlenvolumen, trockenes Ohr, Selbstreinigung

Messgrößen: Otorrhoe, Infektionen, Arztbesuche, Schwindel, HRQOL

Eine gute „Höhle“ ist möglichst klein, überschaubar und selbstreinigend [30-32].

Die Anlage einer offenen Mastoidhöhle durch Wegnahme der hinteren Gehörgangswand (engl. canal wall down, CWD) ist eine oft notwendige und vorgenommene Praxis in der sanierenden Ohrchirurgie. Unabhängig von einer präferierten Operationsstrategie wird, wenn möglich, die entstandene Kavität im gleichen Eingriff oder später obliteriert. Erste technische Beschreibungen verwendeten Knochenspäne und Knochenmehl zur Obliteration
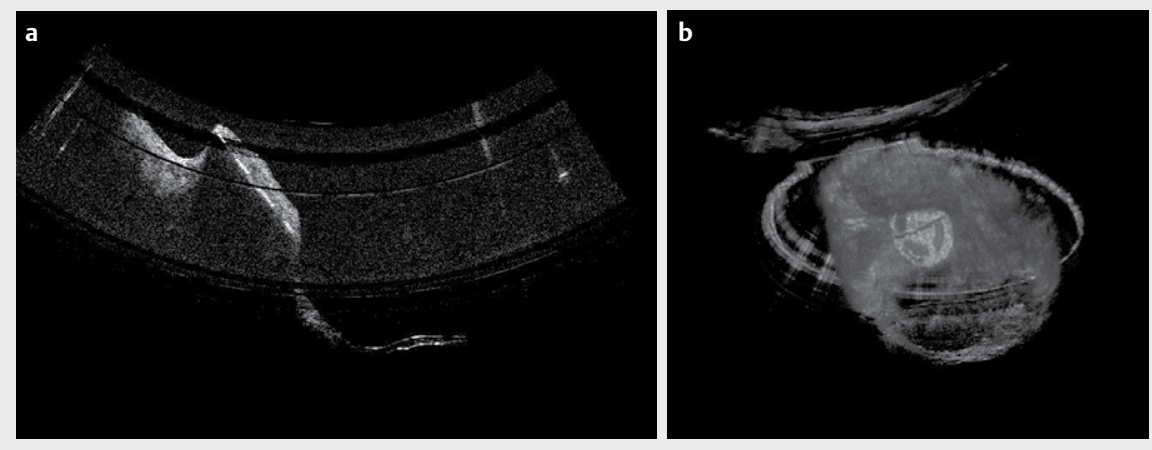

- Abb 1 Optische Kohärenztomographie (OCT) zur Darstellung des Trommelfells. Dargestellt ist der optische zweidimensionale Schnitt durch die Trommelfellebene im hinteren oberen Quadranten. a Der Prothesenteller (2,5 mm Titan Clip Prothese, Typ Dresde, Fa. Kurz, Dusslingen) ist im Längsschnitt gut erkennbar. b In der 3-dimensionalen Rekonstruktion ist der Prothesenteller gut erkennbar. Die Schwingungsanalyse (hier nicht dargestellt) erlaubt Aussagen über die Amplitude des Trommelfells und des Prothesentellers. 
[33, 34]. Zahlreiche Gründe sprechen für die Obliteration offener Mastoidhöhlen. Für die Patienten stehen unaufwendigere Nachsorgen aufgrund der Selbstreinigungstendenz [35-37] und weniger thermische Nebenwirkungen durch Wind, Wasser und saugende Reinigungsmanöver [38] im Vordergrund. Audiologisch erzielen obliterierte Mastoidhöhlen bessere Ergebnisse, da die Schallübertragung bei offener Höhle und damit maximal erweitertem Gehörgang akustomechanisch zu einem geringen Schalldruck vor dem Trommelfell führt [39-41]. Dies resultiert in bis zu 10dB schlechteren Hörergebnissen [37, 42, 43]. Zudem spielen obliterierte Mastoidhöhlen bei der Druckregulation im Mittelohr keine Rolle mehr und haben damit auch keinen negativen Einfluss durch die resultierende Schleimhaut-Oberflächenverkleinerung [44-46]. Aus diesem Grund werden Obliterationen auch bei Erhalt der hinteren Gehörgangswand durchgeführt [47-49]. Nicht zuletzt sind ökonomische Vorteile einer erfolgreichen Obliteration zu nennen, da weniger Arztbesuche und seltener lokale Behandlungen oder gar Revisionsoperationen nötig sind [50,51].

Eine erfolgreiche und stabile Mastoidobliteration ist daher als Qualitätsindikator für eine sanierende Ohroperation zu werten und Obliterationstechnik wie Materialauswahl beeinflussen direkt das Ergebnis. Heute stehen neben den autologen eine Reihe von alloplastischen Materialien zur Verfügung, die insbesondere bei Revisionsoperation und im Falle von biologisch minderwertigem körpereigenem Gewebe deutliche Vorteile besitzen. Bindegewebige Obliterationen [33] oder Fett [52,53] unterliegen aufgrund von Resorptionsvorgängen oft erhebnlichen Volumenreduktionen, was den Obliterationseffekt zunichtemacht [54-56]. Die Verwendung von Muskel-Faszien-Bindegewebslappen, vorrangig aus dem M. temporalis geformt [55, 57-63], haben eine verringerte Schrumpfungstendenz, langfristig können partielle Atrophie und Volumenminderung jedoch nicht vermieden werden [51, 63-65].

Weitere körpereigene biologische Gewebe sind Knochen in Form von Knochenmehl (auch Bohrmehl oder engl. bone pâté, bone dust) oder Chips [54, 64, 66-74] bzw. Knorpel aus Tragus und/ oder dem Cavum conchae [75-78], die während der Operation gewonnen werden. Bei der Verwendung von autologem Knochen wird die Erfolgsrate einer dauerhaften Obliteration entscheidend von den Entnahmeparametern und der Spenderkonstitution beeinflusst. Der mittels Fräse gewonnene Knochen wird in eine pastenartige Mischung aus Zellen, Kollagenbestandteilen, Wasser, Blut und extrazellulärer Matrix zerlegt, in der die Menge an vitalen Zellen über die Fähigkeit der Mineralisierung entscheidet. Jede Kontamination mit Cholesteatomgewebe muss vermieden werden. Durch die Gewinnung mittels Fräse in einem zerspanenden Verfahren entstehen je nach Fräsengeometrie (Durchmesser und Schneidenabstand) unterschiedlich große Knochenspäne. Anpressdruck, Umdrehungsgeschwindigkeit und Kühlung bedingen durch die resultiere Hitze zudem den Anteil vitaler Zellen im Knochenmehl. Große $(7,0 \mathrm{~mm})$ und grobe Fräsen, die nicht mit mehr als 15000 $\mathrm{U} / \mathrm{min}$ verwendet werden, zeigen bei histologischen Untersuchungen den höchsten Anteil vitaler Zeller im nativen Knochenmehl [79]. Alternativ können größere Knochenpartikel auch gesammelt und einer Knochenmühle zerkleinert werden. Tierexperimentell weisen Obliterationen von Defekten nicht-kritischer Größe mit schonend gewonnenem autologem Knochen 2 Wochen nach Operation radiologisch wie histologisch die beste osteogene Durchset- zung auf [80]. Da auch Spender-spezifische Faktoren wie Alter, Hormonstatus und Stoffwechselerkrankungen die Qualität des autologen Knochentransplantats negativ beeinflussen, können auch bei sorgfältiger und kontrollierter Knochenmehlgewinnung partielle Abstoßungen bzw. Resorptionen nicht sicher vermieden werden [71, 72, 81-85].

Die Gewinnung eines qualitativ hochwertigen Materials für die autologe Knochenmehlobliteration setzt daher kontrollierte Drehzahlen bis maximal $15000 \mathrm{U} /$ min bei großem Fräsendurchmesser und grober Scheidengeometrie voraus. Die Beimengung von Antibiotika in das Knochenmehl vor der Reimplantation kann das Infektionsrisiko reduzieren. Unmittelbar postoperative Infektionen des eingebrachten Knochenmehls können durch eine unsachgemäße Gewinnung mit großen Anteilen avitalen Gewebes, oder einer ungeeigneten Entnahmestelle zurückzuführen sein. Kortikaler Knochen bietet, wohl aufgrund seines höheren Zellgehalts, bessere Voraussetzungen für die Obliteration. Walker und Kollegen konnten so die postoperative Infektionsrate von $10 \%$ (9/90) auf 3,6\% (7/195) senken [86].

Körpereigener Knorpel kann alleinig oder in Kombination mit Knochenmehl oder anderen Materialien eingesetzt werden. Die biologischen und mechanischen Eigenschaften machen seinen Einsatz als stabiles Rekonstruktionsmaterial oder als flexible, aber dennoch dichte Abdeckung von zusätzlich verwendetem Obliterationsmaterial. Die Entstehung von prominenten Kanten und Stufen muss zur Verhinderung von Plattenepithelinvaginationen vermieden werden [51].

Alloplastische Materialien konkurrieren im OP-Saal naturgemäß mit autologen Materialien, deren Biokompatibilität, Verfügbarkeit, Kosteneffizienz und Akzeptanz bei Patient und Chirurg unbestritten sind. Daher müssen alloplastische Materialien in der Gesamtschau deutliche Vorteile bieten, die ihre Verwendung attraktiv machen. Verwendung finden u. a. Keramiken [81, 83-85, 87-97], Methylmethacrylat [98], Silikon [99], Hydroxylapatit [58], und Bioaktives Glas (BAG S53P4; BonAlive ${ }^{\circledR}$ )[100-103].

Misst man den Erfolg der Obliteration an der postoperativen Entzündungskontrolle, respektive der Otorrhoe, so können bis zu $97 \%$ ( $n=37 / 38$ ) beschwerdefreie Ohren nach Obliteration mittels Knochenmehl und Knorpel [87] erzielt werden. In einem retrospektiven, direkten Vergleich von obliterierten und nicht-obliterierten Mastoidhöhlen nach Resektion der hinteren Gehörgangswand konnten Harun und Mitarbeiter nach 6 Monaten trockene Verhältnisse in $77,8 \%(14 / 18)$ und $71,1 \%(41 / 45)(p=0,590)$ erzielen. Im weiteren Verlauf stiegen diese Werte auf 88,9\% (16/18) und 91,1\% (41/45) $(p=0,786)$ an, so dass hier insgesamt kein Vorteil einer Obliteration hinsichtlich des „trockenen Ohres“ belegt werden konnte. Auch nach Stratifizierung in primäre und sekundäre Obliterationen konnte kein signifikanter Unterschied erzielt werden [104]. Auch die Anzahl der postoperativen Arztkonsultationen zeigte keinen Unterschied in den beiden Gruppen.

Ein weiterer für den Patienten wichtiger Aspekt ist die Schwindelkontrolle. Hier haben die Obliterationen einen eindeutig positiven Effekt, nach dem in bis zu 56 \% nach der Obliteration kein Schwindel bei alltagsbedingter thermischer Reizung auftritt $[38,66]$.

Dass die Patienten durch die Obliteration einen Benefit verspüren, lässt sich mittels Glasgow Benefit Inventory (GBI) erfassen 
[36, 105-108] (• Abb. 2). Dieses Messinstrument zur Nutzenbewertung nach HNO-Eingriffen wird im Kapitel 3.7 vorgestellt.

\subsection{Rezidivrate (Cholesteatomrezidive, -residuale)}

Ziele: $\quad$ Eradikation der Erkrankung

Messgrößen: Residual- und Rezidiv-Rate

Die Frage nach „der richtigen“ Strategie oder Operationsmethode in der Cholesteatom-Behandlung hat in den vergangenen Jahren eine intensive Bearbeitung in der Literatur erfahren. Problematisch gestaltete sich hierbei, dass in der Nomenklatur von Techniken Unschärfen bestanden. Die Definition anhand des Zustandes der erhaltenen (engl. canal wall up, CWU) und weggenommenen (engl. canal wall down, CWD) hinteren Gehörgangswand hat sich über die Jahre durchgesetzt. Zudem wird die neue Klassifikation der Tympanomastoid-Chirurgie hoffentlich noch mehr Schärfe in die Definition von Vorgehen und Ausprägung der chirurgischen Technik bringen [109].

Die umfassendste Untersuchung stellt aktuell die Meta-Analyse aus dem Jahr 2013 dar. Dabei wurden 13 Studien bewertet (4720 Patienten; 2761 CWU und 1959 CWD) [110]. Im Ergebnis reichten die Rezidivraten von 9 bis $70 \%$ in der CWU und 5 bis $17 \%$ in der CWD Gruppe. Daraus ergab sich ein fast 3-fach höheres Risiko ein Rezidiv zu erleiden, wenn die hintere Gehörgangswand intakt belassen wurde (CWU) in Vergleich mit der CWD-Gruppe. Die Limitationen der Studie und zusätzliche Einflussfaktoren wurden von den Autoren intensiv diskutiert. Insbesondere die Unterschiede im Nachbeobachtungszeitraum, die oftmals fehlende Unterscheidung in Rezidiv- bzw. Residual-Cholesteatome und die Durchführung von 2nd look Operationen. Abschließend kommen die Autoren jedoch zu der Empfehlung, dass die CWD-Technik großzügiger angewendet und letzten Endes auch bevorzugt werden sollte. Daraus leitet sich auch die Empfehlung eines mindestens 2- eher 5-jährigen Intervalls zur abschließenden Beurteilung von Cholesteatom-Rezidiven (-Residuen) ab [35, 109-111].
Besonderes Augenmerk gilt es der einzeitigen Obliteration bei CWD-Technik zu widmen, da die Gefahr einer Versprengung von Cholesteatom-Gewebe in die Obliteration besteht. Hinsichtlich des Auftretens von Residual- oder Rezidiv-Cholesteatomen scheint sich die einzeitige Mastoid-Obliteration jedoch positiv auszuwirken [114]. Im systematischen Vergleich von 13 Studien mit insgesamt 1534 Ohren konnte eine Rezidivrate von 4,6\% (0-12\%) und eine Residualrate von 5,4\% (0-12,5\%) bei obliterierten Mastoidhöhlen identifiziert werden, unabhängig von einer offenen (CWD) oder geschlossenen (CWU) Technik. Demgegenüber stehen Rezidiv- und Residualraten von 4 bis $17 \%$ bei offener Operationstechnik (CWD) und 9 bis $70 \%$ in Studien mit geschlossener Operationstechnik [104]. Ob eine Verwendung autologen oder alloplastischen Materials einen Unterschied im Auftreten von Residual- oder RezidivCholesteatomen macht, kann noch nicht abschließend beurteilt werden. Die Residualraten waren zwar nahezu identisch bei 5,5\% ( $n=73$; autologe Obliteration) und 4,7\% ( $n=10$; alloplastische Obliteration), während die Rezidivraten bei 5,3\% ( $n=70$; autologe Obliteration) und $0,5 \%(n=1$; alloplastische Obliteration) lagen. Allerdings lässt die Anzahl der alloplastisch obliterierten Höhlen (212 Patienten in 3 Studien $[110,115,116])$ einen Bias vermuten. Zusammenfassend lässt die derzeitige Studienlage den Schluss zu, dass eine einzeitige Mastoidobliteration im Rahmen der Cholesteatom-Operation die Residual- und Rezidivrate, im Vergleich zur zweizeitigen, nicht beeinflussen.

\subsection{Hörergebnisse}

Die Dokumentation von Hörergebnissen nimmt naturgemäß bei der Ergebnisbeschreibung von Mittelohroperationen eine zentrale Rolle ein. Daher haben sich audiologische Ergebnisse als Qualitätsindikatoren in der Mittelohrchirurgie bewährt und international durchgesetzt. Die Qualität der Therapie kann zum Teil an der Änderung der Hörleistung gemessen werden. Der primär durch eine Mittelohroperation beeinflussbare Anteil der Schwerhörigkeit ist

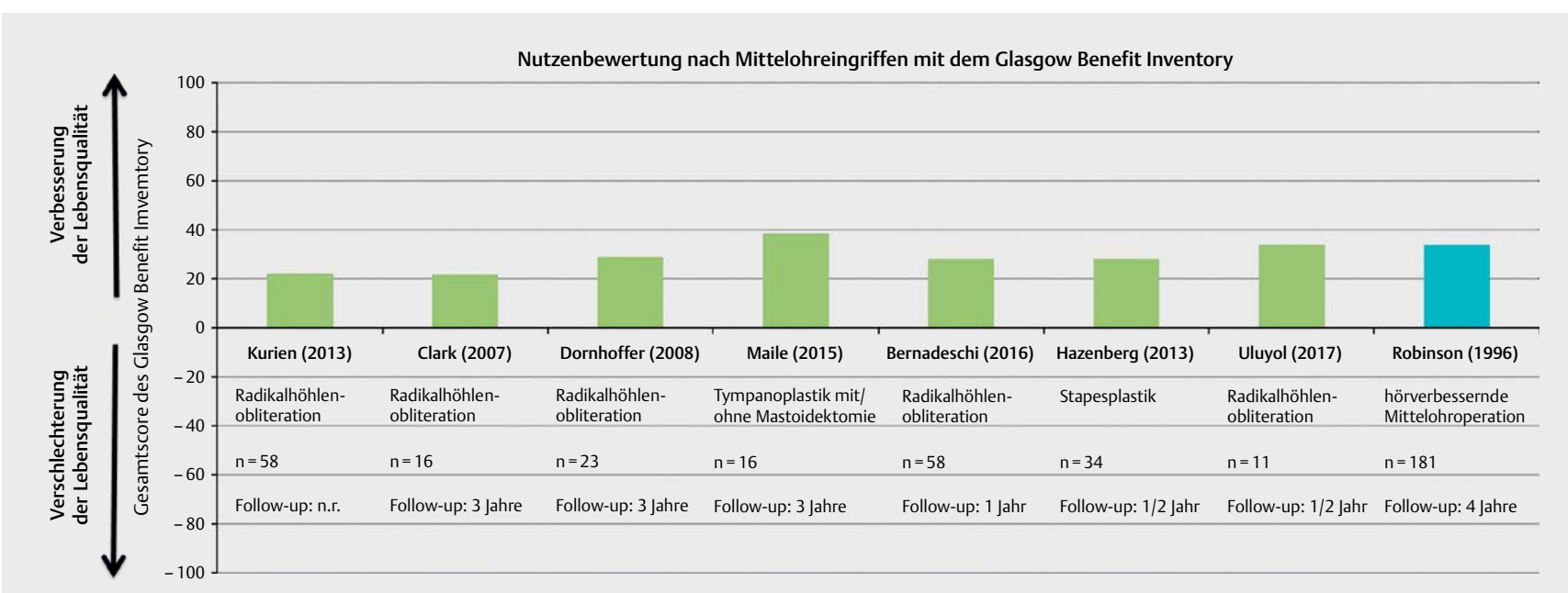

Abb. 2 Nutzenbewertung nach Mittelohreingriffen bewertet mit dem Glasgow Benefit Inventory. Der Glasgow Benefit Inventory (GBI) trägt Verbesserungen nach oben und Verschlechterungen (jeweils auf maximal 100 Punkte skaliert) auf. In den dargestellten Studien konnte jeweils ein positiver Nutzen nach durchgeführter Intervention von den Patienten angegeben werden [35, 36, 105, 107, 108, 140, 199, 200]. 
die Schallleitungsschwerhörigkeit, bei deren Beschreibung die folgenden Ziele verfolgt werden (modifiziert nach[117]):

1. Möglichkeit der Erfolgsprognose für Patient und Arzt

2. Beurteilung einer Operationsmethode oder einer Rekonstruktionstechnik

3. Vergleich der Ergebnisse mit anderen Fallserien und Studien

4. Schaffung einer Datengrundlage für weiterführende MetaAnalysen.

Qualitativ hochwertige Berichts- und Dokumentationsstandards müssen, um national und international vergleichbar und anschlussfähig zu sein, folgende Voraussetzungen erfüllen:

1. Anwendbarkeit: Bei der Definition der Parameter muss auf die Verhältnismäßigkeit von gewünschtem Erkenntnisgewinn und zeitlich wie ökonomisch machbarer Umsetzung geachtet werden. Dies erhöht die Akzeptanz und die Anwendung eines Dokumentationsstandards.

2. Validität: Die definierten Parameter müssen den Erfolg der Intervention nachgewiesenermaßen abbilden. Dies betrifft insbesondere psychometrische Messinstrumente.

3. Vollständigkeit: Nach Möglichkeit sollten alle Parameter erfasst werden, die einen Einfluss auf den Interventionserfolg haben und/oder ihn widerspiegeln. Dies erfordert eine Kombination von Bewertungskriterien und Messmethoden (anamnestische, klinische und intraoperative Befunde, Funktionsergebnisse).

4. Übertragbarkeit: Die zur Erfolgsbeschreibung herangezogenen Parameter sollten die international verwendeten Parameter beinhalten, um sie im internationalen Kontext diskutieren zu können. Dies trifft für Messmethoden, -instrumente und Standards in der Ergebnisberechnung, bzw. -darstellung zu.

5. Vergleichbarkeit: Die Studienpopulationen müssen möglichst genau beschrieben sein, damit Vergleiche der Erfolgsparameter nicht durch zu große Unterschiede in der Kohortenzusammensetzung entkräftet werden.

Zur Bewertung der Hörveränderung nach Mittelohreingriffen wird in den meisten Fällen die Veränderung der Schallleitungsschwerhörigkeit herangezogen. Diese ist mittels Reintonaudiometrie leicht ermittelbar. Das Reintonaudiogramm stellt somit nach wie vor das wichtigste psychoakustische Messinstrument dar. Seine intuitiv interpretierbare Aussage in Form von Luft- und Knochenleitungshörschwellen (in dB) ist leicht darstellbar, auszuwerten und einem mathematischen Vergleich zugänglich. Sein tonaler Charakter erlaubt darüber hinaus auch vergleichende Aussagen über Sprachgrenzen hinweg, was das Reintonaudiogramm in der internationalen Literatur unersetzlich macht. Auch aufgrund seiner nachgewiesenen Validität wird es als Korrelationsgrundlage für andere Outcome-Parameter herangezogen [118]. Die Sprachaudiometrie weist für die Nutzenbewertung in der Mittelohrchirurgie einige Besonderheiten auf, die ihren Einsatz erläuterungswürdig machen.

\subsubsection{Reintonaudiometrie}

Die Bestimmung der Differenz von Knochen- und Luftleitungshörschwelle (engl. air-bone gap, ABG) ist der durch eine Tympanoplastik beinflussbare Aspekt der Hörstörung. Messtechnisch sind die beiden Schwellen in der Regel leicht zu ermitteln und somit der ABG, gemittelt über die verwendeten Prüffrequenzen, nicht nur rasch berechnet, sondern ein zentraler Parameter der bei der Qualitätsbewertung. Eine unkritische Verwendung verbietet sich dennoch, weil eine Abnahme des ABG auch durch einen postoperativen Anstieg der Knochenleitungshörschwelle („Knochenleitungsabfall“) bei unveränderter Luftleitungshörschwelle (engl. air conduction, AC) entstehen kann [117-119].

Um diese falsch positive Abnahme des AGB auszuschließen müssen entweder zusätzlich die Veränderung der Knochenleitungshörschwelle (engl. bone conduction, BC) im prä-post-OP-Vergleich angegeben werden, oder aber zusätzlich eine Rechenoperation durchgeführt werden, bei der von der postoperativen Luftleitungshörschwelle die präoperative Knochenleitungshörschwelle subtrahiert wird $\left(A B G_{\text {eff }}=A C_{\text {post }}-B C_{\text {prä}}\right)[122]$.

Weiterhin ist zu berücksichtigen, dass für den Patienten die Veränderung der Luftleitungshörschwelle von entscheidender Bedeutung ist, stellt sie für ihn doch die Nettoleistung der hörverbessernden Operation dar. Somit muss bei der Formulierung von Qualitätsindikatoren, neben dem chirurgisch interessanten $A B G$ auch die resultierende Luftleitungshörschwelle aus Sicht des Patienten berücksichtigt werden, da sie auch die resultierende Lebensqualität stark beeinflusst.

Die Auswahl der Prüffrequenzen sollte sich zur internationalen Vergleichbarkeit von Ergebnissen nach den Empfehlungen der amerikanischen Fachgesellschaft (American Acadamy of Otolaryngology - Head and Neck Surgery, AAO-HNS) richten und folglich 0,5; 1; 2 und $3 \mathrm{kHz}$ beinhalten [123]. Angegeben werden Mittelwert und Standardabweichung des Reintonmittelwerts (engl. pure-tone average, PTA). Im deutschsprachigen Raum sind anstelle von 3 eher $4 \mathrm{kHz}$ verbreitet. In den Zeiten der digitalen Datenverarbeitung sollte die Angabe beider Mittelwerte, sowie einer über alle gemessene Frequenzen gemittelten Wertes, problemlos möglich sein. Da die Auswahl der berücksichtigten Frequenzen einen Einfluss auf die Ergebnisse hat [124], ist in jedem Fall die getroffene Wahl derselben mit anzugeben.

\subsubsection{Sprachaudiometrie}

Die Ergebnisse der Sprachaudiometrie sind zur Beurteilung der Schallleitungsschwerhörigkeit von eingeschränktem Nutzen. Ein dem ABG der Reintonaudiometrie vergleichbarer Parameter der Sprachaudiometrie existiert bisher nicht. Ähnlich der Luftleitungshörschwelle fassen die die sprachaudiometrischen Ergebnisse multiple Teilaspekte des Hörens zusammen, die jedoch für die Bewertung der Funktionseinschränkung bzw. der -wiedererlangung bedeutend sind [125].

Die international bestehenden methodischen Unterschiede und Bewertungsdifferenzen lassen direkte Vergleiche nicht zu. Auch der im anglo-amerikanischen Raum bevorzugte Wort-Verständnis-Wert (engl. Word Recognition Score, WRS), gemessen bei $40 \mathrm{~dB}$ über der individuellen Sprachverstehensschwelle [126, 127], muss als methodisch unbrauchbar eingestuft werden. Bei individueller und damit variabler Festlegung des Darbietungsschalldrucks wird aufgrund und Ausmaß der vorliegenden Schwerhörigkeit entweder die Unbehaglichkeitsschwelle bzw. die Pegelgrenze des Audiometers erreicht oder die Ergebnisse entsprechen nicht dem maximalen Sprachverstehen $[128,129]$. Demgegenüber haben die Veränderungen des Sprachverstehens bei konstanten Schalldruckpegeln signifikant größere Unterschiede geliefert, sodass konkret die Angabe des prozen- 
tualen Freiburger Einsilber-Verstehens bei 65 und $80 \mathrm{~dB}$ als methodisch überlegen angesehen werden muss [128].

Somit ist die Einbeziehung sprachaudiometrischer Ergebnisse zur Qualitätsbeschreibung von hörverbessernden Mittelohroperationen prinzipiell wünschenswert, allerdings sind die nationalen wie internationalen methodischen Differenzen für die Aussagen limitierend. Insbesondere muss vor diesem Hintergrund die 2012 erfolgte Modifikation der AAO-HNS Empfehlungen zum Berichtsstandard [130] äußerst kritisch bewertet werden. Danach sollen Hörergebnisse immer als 2-dimensionale Parameterkombination aus Ton- und Sprachaudiometrie-Ergebnissen (sog. Verteilungsdiagramm, engl. scattergramm) dargestellt werden. Die o.g. Ausführungen lassen jedoch allein bereits die methodische Vorbedingung (Messung bei $40 \mathrm{~dB} \mathrm{SL}$ ) als nicht geeignet erscheinen, um Veränderungen durch hörverbessernde Operationen verlässlich abbilden zu können [128]. Zudem stellt sich die Frage, wie anhand des propagierten Scattergramms eine Fallzahlschätzung für klinische Studien erfolgen soll. Seine zudem verpflichtende Nutzung als Voraussetzung für Publikationen in einigen Zeitschriften muss daher als bedenkenswert eingestuft werden.

\subsubsection{Erhebungszeitpunkte}

Neben der obligaten präoperativen Messung sind die Zeitpunkte der postoperativen Erhebung der audiometrischen Ergebnisse wenig standardisiert. Die AAO-HNS Empfehlungen sehen ein Intervall von 12 Monaten für aussagekräftige Hörergebnisse vor [123]. Ein Konsens über definierte Zeitintervalle, die Kurz- von Langzeitergebnissen unterscheiden, existiert nicht. Zeitintervalle $>36$ bis $>60$ Monate können jedoch, ähnlich wie in der CholesteatomChirurgie [37,111-113], relativ sicher als Langzeitaussage gewertet werden.

In der klinischen Routine sind die ersten Wochen und Monate nach der Operation - auch audiometrisch - am besten dokumentiert, was an der vergleichsweise engen Bindung des Patienten an den Operateur in diesem Zeitraum liegt. Daher existieren oft Funktionsüberprüfungen zum Zeitpunkt der Detamponade bzw. kurz danach. Durch die noch nicht abgeschlossene Wundheilung, ist die Belastbarkeit der Ergebnisse jedoch gering und eher schlechter als abschließend zu erwarten. Ein Intervall von 3 Monaten scheint aus der klinischen Erfahrung heraus praktikabel zu sein. Wie bei jedem anderen Parameter auch, ist in jedem Fall die Angabe des berichteten Zeitintervalls unerlässlich.

Wie so oft scheitern die Bemühungen um eine lückenlose Dokumentation leider allzu oft an den Gegebenheiten der Versorgungsrealität. Wie im Abschnitt „Erfassungs- und Dokumentationssysteme“ ausgeführt werden wird, ist die Menge an „missing data" der Länge des postoperativen Beobachtungszeitintervalls direkt proportional. Dieses Phänomen lässt sich auch nur bedingt durch gesteigerte Anstrengungen des Operateurs in Praxis oder Klinik beheben, da die Compliance der Patienten ihrerseits direkt proportional zum Beschwerdeausmaß, respektive dem Leidendruck ist [8]. Dies führt zudem zu einer Verzerrung im Datenpool hin zu einer überrepräsentierten Abbildung von besonders langwierigen und ggf. komplikationsbehafteten Verläufen. Die betroffenen Patienten verbleiben länger in der klinischen Beobachtung und erhalten häufiger Funktionskontrollen, als ihre regulär genesende Vergleichsgruppe.

\subsection{Lebensqualität}

Die Zahl an Lebensqualitätsmessinstrumenten, mit denen die Beeinträchtigung von Erkrankten und der Therapieerfolg von behandelten Ohrpatienten gemessen werden kann, ist beachtlich und weiter steigend [131]. Auch wenn die Fülle der beschriebenen Bewertungsinstrumente auf den ersten Blick unnötig erscheint, so ist sie für eine differenzierte Beurteilung dennoch erforderlich. In der Vergangenheit wurden häufig selbsterstellte Fragelisten für Patienten und Symptomdokumentationslisten als Ergänzung zu objektiven oder funktionellen Parametern herangezogen [131-135]. Die Bemühungen, mit diesen Listen eben solche Faktoren zu erfassen und messbar zu machen, die sich nicht in $A B G$ oder prozentualen Heilungsraten abbilden lassen, sind nachvollziehbar und korrekt. Allerdings erfüllen sie nicht die Gütekriterien wissenschaftlicher Messinstrumente und haben daher lediglich ergänzenden Charakter.

Daher war die Einführung validierter Messinstrumente konsequent und ist für die Beschreibung der Ergebnisqualität eine Bereicherung. Für die Ohrchirurgie war im Jahr 2000 mit dem „Chronic Ear Survey (CES)“ erstmals ein Ohr-spezifisches Messinstrument in englischer Sprache zur Lebensqualitätsmessung verfügbar [136]. Der COMOT-15 (Chronic Otitis Media Outcome Test 15) war im Jahr 2009 das erste deutsche Ponton [132].

\subsubsection{Generische und spezifische Messinstrumente}

Im Vergleich zu generischen Lebensqualitätsmessinstrumenten fokussieren krankheitsspezifische auf eben die körperlichen Symptome und psychischen Beeinträchtigungen, die durch eine bestimmte Erkrankung entstehen. Daher benötigt man für die strukturierte Erfassung der Beschwerden bei der Otosklerose [137] auch ein anderes Frageninventar als bei der chronischen Otitis media. Ferner erklärt diese Spezifität auch, warum Ohr-erkrankungsbedingte Beein-

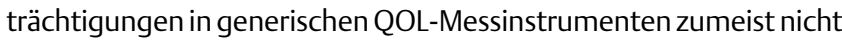
abgebildet werden können, da sie zu umfassend ausgerichtet sind.

Zu den in der HNO-Heilkunde krankheitsübergreifend eingesetzten generischen Messinstrumenten zählt der Short Form 36 (SF-36) und der GBI. Auch wenn der SF-36 in den bisherigen Studien zu Mittelohroperationen keine Verbesserung der HRQOL belegen konnte, weisen doch Patienten mit einer chronischen Otitis media signifikante Einschränkungen in 4 Subskalen (von insgesamt 8) auf [138-140]. Insbesondere vor dem krankheitsübergreifenden Charakter auch jenseits des HNO-Fachgebiets ist die zusätzliche Verwendung des SF-36 zur Bewertung bei Mittelohroperationen zu empfehlen. Somit sind unter Umständen eine Einordnung von Mittelohr-spezifischen Einschränkungen und/oder Veränderungen nach der Therapie nicht nur longitudinal, sondern auch Entitäten übergreifend möglich.

Der GBI wurde ebenfalls als Krankheits- und Therapie-übergreifendes Messinstrument entwickelt, um den allgemeinen Nutzen von HNO-Eingriffen zu messen [140]. Ursprünglich war die Darstellung der Ergebnisse mit Zahlenwerten von -100 (starke Verschlechterung) bis +100 (starke Verbesserung) vorgesehen, um die relative Veränderung durch die Intervention abzubilden. Oft werden die mittels CBI gemessenen Daten jedoch mit den statistischen Maßen von Mittelwert und Standardabweichung angegeben. Ungeachtet dessen eignet er sich ebenfalls, um den Nutzen eines, bzw. die Veränderung durch einen mittelohrchirurgischen Eingriff abzubilden und zusätzlich Vergleiche zu GBI-Messungen bei anderen Interven- 
tionen zu ziehen. Nicht unerwähnt soll bleiben, dass die deutsche Version des GBI bisher nicht für die Nutzenmessung nach Tympanoplastik validiert ist. Einsatz fand der GBI in der Mittelohrchirurgie bisher bei der Bewertung von Radikalhöhlenrevisionen und Stapes-Operationen. Die $>$ Abb. $\mathbf{2}$ stellt Studien, in denen der Nutzen mittels $\mathrm{GBI}$ gemessen wurde nebeneinander.

\subsubsection{HRQOL-Messinstrumente in der Mittelohrchirurgie}

Bei der Nutzenbewertung von Mittelohreingriffen kann zusätzlich zwischen der Bewertung der Sinnesqualität „Hören“ und den krankheitsspezifischen Beeinträchtigungen unterschieden werden. Einige der krankheitsspezifischen Messinstrumente haben nochmals Subskalen zur Hörbewertung integriert.

Die Bewertung des Hörverlusts bzw. einer postinterventionellen Änderung ist Gegenstand der Evaluation von operativen Verfahren, wie der Tympanoplastik, aber auch der Hörsystemversorgung. Messinstrumente, die speziell den Hörverlust bewerten sind in der Vergangenheit vorrangig eingesetzt worden, um den Nutzen von konventionellen Hörgeräteversorgungen sowie Hörimplantaten abzubilden. Ihre Stärke liegt darin, dass sie eine Erfassung der tatsächlichen, individuellen Beeinträchtigung erlauben, die durch eine Höreinschränkung besteht. Somit ergänzen sie in besonderem Maße die psychophysischen Testverfahren und sind nicht als Surrogat-Parameter zu betrachten. In den letzten Jahren wurden einige bereits eingesetzt, um die Hörverbesserung nach Mittelohreingriffen zu bestimmen.

Zur reinen Bewertung des Hörverlusts stehen u. a. die folgenden Inventare und Messinstrumente zur Verfügung:

- Hearing Satisfaction Scale (HSS)

- (modified) Amsterdam Inventory of Auditory Disability and Handicap ((m)AIAD)

- Hearing Handicap Inventory for Adults (HHIA)

- Abbreviated Profile of Hearing Aid Benefit (APHAB)

Zudem lässt sich die empfundene Klangqualität über

- die Amsterdam Post Operative Sound Evaluation (APOSE) erfassen.

Um die subjektive Beeinträchtigung durch eine spezifische Ohrerkrankung messen zu können, ist die Verwendung von multidimensionalen krankheitsspezifischen Bewertungsinstrumenten erforderlich. Wie alle bisher beschriebenen Messinstrumente auch, ist ihr Einsatz zur Beschreibung des Ist-Zustands möglich. Dies macht sie zu einem wertvollen Instrument in der klinischen Routine. Die entsprechenden Item-Listen fragen die speziellen Symptome der jeweiligen Erkrankung ab und stehen somit in engem Zusammenhang mit der systematischen ärztlichen Anamnese, ergänzen diese jedoch durch die Dimension der subjektiven skalierten Bewertung der Beeinträchtigung.

Zusätzlich besteht die Möglichkeit des individuellen longitudinalen Einsatzes zum Vergleich der prä-/post-therapeutischen Veränderung. Analog zur Ergebnisbewertung des Operationserfolges einer hörverbessernden Operation anhand der ABG-Abnahme kann somit die subjektive Beeinträchtigung durch die Symptome der Krankheit be- und ausgewertet werden.

Die $>$ Abb. 3 gibt einen Überblick der zur Verfügung stehenden Messinstrumente, die zur Erfassung der subjektiven Beeinträchti- gung bei Mittelohrerkrankungen eingesetzt werden können und sich gleichzeitig zur Nutzenbewertung von therapeutischen Maßnahmen im Verlauf eignen ( $\triangleright$ Abb. 4).

\subsubsection{Weitere Einflussfaktoren auf die HRQOL}

Die Beobachtung, dass Patienten das Ergebnis ihrer Operation unterschiedlich bewerten trotz gut vergleichbarem objektivem Outcome lässt die Frage nach zusätzlichen, ggf. übergeordneten Einflussfaktoren aufkommen. Insbesondere gegenläufige Bewertungen mit guten Werten der objektivierbaren Ergebnisparameter (verschlossenes Trommelfall, trockenes Ohr und ABG $<10 \mathrm{~dB}$ ) und dennoch stark eingeschränkter spezifischer Lebensqualität legen die Vermutung nahe, dass HRQOL Messungen einem intraindividuellen Bias unterliegen. In einer Untersuchung zum Einfluss der psychischen Gesundheit auf die krankheitsspezifische HRQOL bei Patienten mit chronischer Otitis media konnte gezeigt werden, dass die Depressivität der Haupteinflussfaktor für den postoperativ empfundenen Nutzen einer Mittelohroperation ist [141].

Einhundert Patienten mit OMC zeigten mit dem COMOT-15 und dem ZCMEI-21 signifikante Verbesserungen im prä-/post-OP-Vergleich bei unveränderter generisch gemessener Lebensqualität (SF36). Bei Stratifizierung nach der präoperativ gemessenen Depressivität war mit allen Messinstrumenten die HRQOL bei den Patienten mit depressiven Symptomen stärker eingeschränkt. Auch nach Adjustierung auf die Änderung der absoluten Hörschwelle, dem Ausmaß der Mittelohrpathologie und den somatischen Begleiterkrankungen blieb dieser statistisch signifikante Zusammenhang bestehen. Somit können präoperative Depressionssymptome als prospektiv mit einer schlechteren krankheitsspezifischen Lebensqualität sechs Monate nach einer sanierenden Mittelohroperation assoziiert angesehen werden ( $\triangleright$ Abb. $\mathbf{3}$ ).

Da nach diesen Ergebnissen Patienten mit gesteigerter Depressivität die postoperative HRQOL signifikant schlechter bewerten (auch unabhängig von somatischen Begleiterkrankungen, dem Ausmaß der Mittelohrpathologie und der postoperativen Luftleitungshörschwelle) wird die Notwendigkeit weiterer Untersuchungen zur Identifikation zusätzlicher Einflussfaktoren, wie etwa Persönlichkeitsmerkmalen, deutlich. Gleichzeitig veranschaulichen die Daten die Komplexität bei der Verwendung psychometrischer Messinstrumente, die - wie andere Messergebnisse auch - immer im Gesamtkontext der Messbedingungen gesehen werden müssen. Zur Beratung der Patienten und für eine realistische Erwartungshaltung bei Patient und Arzt können diese Zusammenhänge einen wertvollen Beitrag leisten.

\subsubsection{Empfehlungen zu Auswahl und Einsatz von HRQOL- Messinstrumenten}

Bisher existieren für die Auswahl und den Einsatz dieser Messinstrumente keine nationalen oder internationalen Empfehlungen. Auswahl und Einsatz sind standort-abhängig und noch nicht flächendeckend verbreitet. Zudem gibt es mittlerweile auch in deutscher Sprache scheinbar konkurrierende Bewertungsinstrumente, wie beispielsweise für die chronische Otitis media der COMOT-15 und der ZCMEI-21. Durch Abweichungen in den beiden Frageninventaren unterscheiden sie sich in der Gewichtung der Aussage, was die Autoren und Anwender in den jeweiligen Arbeiten hervorheben und für ihre Anwendungsfälle als vorteilhaft bewerten. Den- 


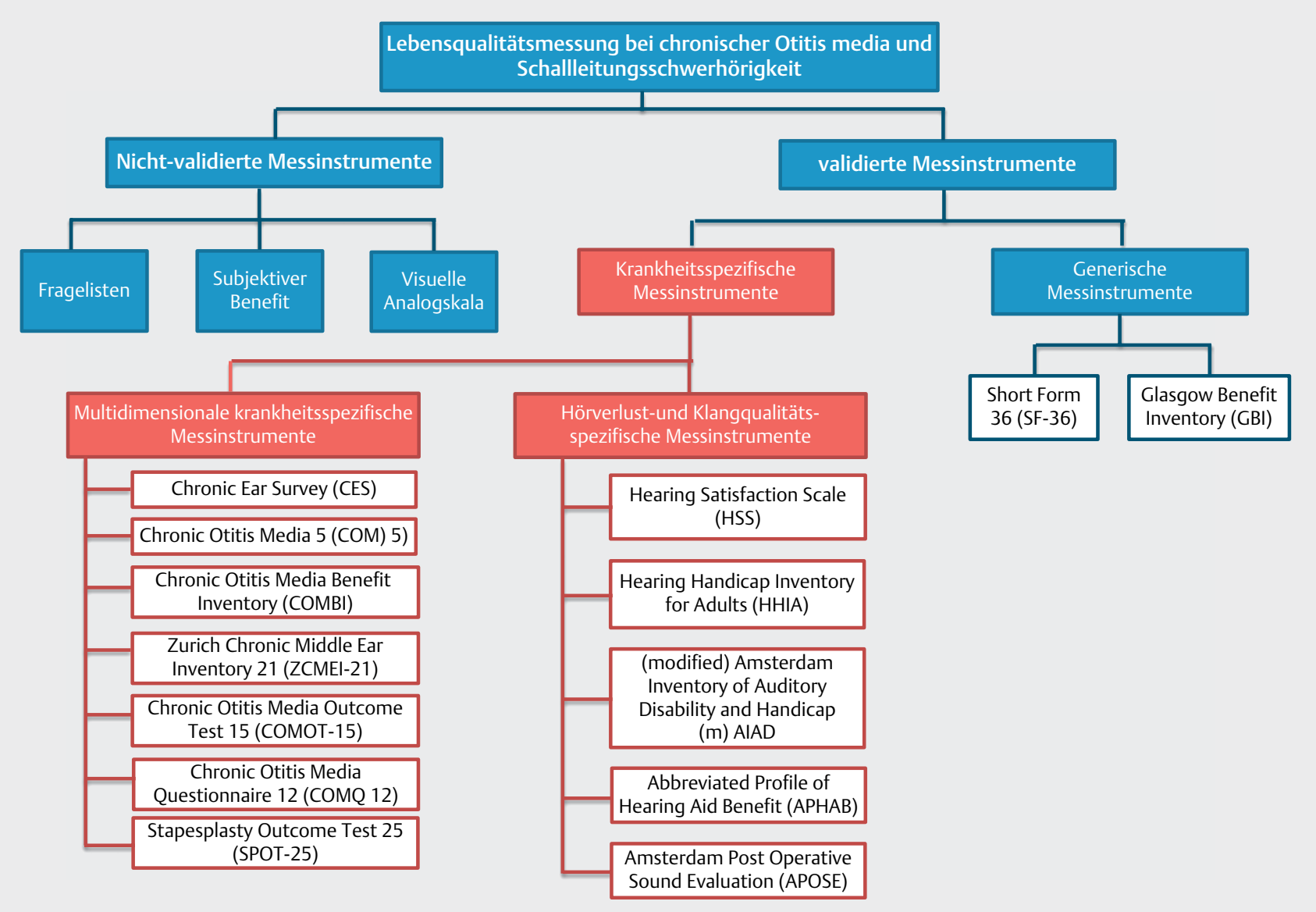

Abb. 3 Messinstrumente zur Nutzenbewertung von Mittelohroperationen

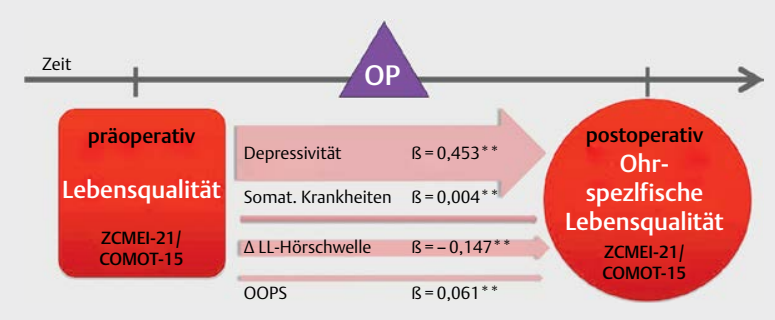

Abb. 4 Beeinflussung der HRQOL durch die psychische Gesundheit. Aufgeschlüsselt nach den Einflussfaktoren stellt die Depressivität den größten Anteil an der postopertiv empfundenen Lebensqualität dar. Die Abnahme der Luftleitungshörschwelle, wie auch die zusätzlichen somatischen Erkrankungen haben einen deutlich geringen Einfluss.. (ZCMEI-21: Zurich chronic middle ear inventory 21; COMOT-15: Chronic otitis media outcome test 15; OOPS: Ossiculoplasty outcome staging index, $\Delta \mathrm{LL}-\mathrm{Hörschwelle:} \mathrm{Veränderung} \mathrm{der}$ Luftleitungshörschwelle)

noch zieht die individuelle Auswahl eines Messinstruments die nicht mehr vorhandene Vergleichbarkeit von Ergebnissen nach sich. Verschärft wird die Situation durch individuelle Übersetzungen von anderen, vorzugsweise englischen Messinstrumenten, die bisher in deutscher Sprache nicht validiert und publiziert sind.
Als otologische Gemeinschaft sehen wir daher einer Entwicklung entgegen, die auf eine wachsende Diversität zustrebt und gleichzeitig einer nationalen wie internationalen Anschlussfähigkeit der Ergebnisse entgegenwirkt. Aus dem Blickwinkel der Qualitätssicherung ist daher dringend die Erarbeitung von Empfehlungen zur Auswahl und dem Einsatz von Lebensqualitätsmessinstrumenten gefordert. Nur so können zukünftig qualitativ hochwertige und national wie international vergleichbare Studienergebnisse produziert werden. Zur Verwendung von HRQOL Messinstrumenten bei der Nutzenbewertung in der Mittelohrchirurgie kann daher festgehalten werden:

- Routinemäßige Verwendung von HRQOL Messinstrumenten zur Bewertung der Beeinträchtigung und Beurteilung des individuellen Behandlungsergebnisses

- Auswahl und Verwendung eines generischen und eines krankheitsspezifischen Messinstruments, welches in möglichst vielen Sprachen validiert ist

- Einsatz prä- und mindestens sechs Monate postoperativ

- Abgleich mit psychophysischen, audiometrischen Messergebnissen (Reintonaudiometrie)

HRQOL-Messinstrumente erweitern die Sichtweise und komplettieren die Krankheitsbewertung eines Individuums durch die Verbindung von spezifischen Beschwerden mit einer mess- und ver- 
gleichbaren Skalierung. Somit wird die Lücke zwischen messbaren Funktionseinschränkungen und subjektiv empfundener, krankheitsbedingter Beeinträchtigung geschlossen [142].

\subsection{Ergebnisqualität als Abwesenheit von Komplika- tionen (ein Paradigmenwechsel)}

Instinktiv liegt der Therapiebewertung die Verbesserung eines eingeschränkten Gesundheitszustandes zugrunde. Auch die vorliegende Arbeit hat sich bisher vorrangig mit Indikatoren unter dem Gesichtspunkt beschäftigt, den Erfolg und die Qualität der Behandlung an deren Verbesserung abzulesen und zu messen. Obgleich jeder erhobene Parameter auch eine Verschlechterung unter der Therapie abzubilden in der Lage ist, wird a priori eine Verbesserung als Ausdruck einer erfolgreichen Behandlung zu recht antizipiert.

Eine grundlegend andere Betrachtungsweise, die einem Paradigmenwechsel in der Bewertung gleichkommt, ist die Definition des Qualitätsbegriffs über die Abwesenheit unerwünschter Nebenwirkungen bzw. Komplikationen. Interessanterweise ist es die Furcht vor eintretenden Komplikationen, die Patienten im Alltag einer Operation kritisch gegenüberstehen lassen. Auch das ärztliche Aufklärungsgespräch widmet sich in erheblichem Maß der Erklärung und realistischen Abwägung der Eintrittswahrscheinlichkeit von Komplikationen. Daher scheint der Perspektivenwechsel nicht nur nachvollziehbar, sondern auch notwendig.

Es existieren ausführliche Arbeiten über Handlungsanweisungen zum Vorgehen beim Auftreten von Komplikationen intra- wie auch unmittelbar postoperativ [143-145]. Schick und Dlugaiczyk haben 2013 in ihrem Referat „Fehler und Gefahren: Ohrchirurgie und Chirurgie der lateralen Schädelbasis“ an gleicher Stelle eine umfassende Arbeit zu den möglichen Komplikationen bei Ohrchirurgischen Eingriffen abgefasst [144]. Diese und andere Arbeiten haben maßgeblich zur Entwicklung eines offenen Umgangs mit Komplikationen und Fehlern beigetragen. In diesem Zug sei auch auf die 2013 erstmals in das Programm des Jahreskongresses 2013 aufgenommene Rubrik „Fehler und Gefahren“ bzw. „Lernen am Fall“ verwiesen.

In der Gesamtheit ist diese Entwicklung uneingeschränkt zu begrüßen. Als Konsequenz aus dieser sich positiv entwickelnden Fehlerkultur darf zusätzlich der Wunsch nach einer routinemäßigen, standardisierten und prospektiven Erfassung von Komplikationen geäußert werden. Denn alle in den Übersichtsarbeiten zitierten Quellen stellen Erfahrungsberichte, Einzelfallbeschreibungen oder retrospektive Analysen von Patientenpopulationen dar. Diese können zur Identifikation möglicher Komplikationen selbstverständlich herangezogen werden und können auch erste Hinweise auf die Auftretenswahrscheinlichkeit bzw. -häufigkeit von Komplikationen geben. In einem nächsten Schritt sind jedoch prospektive Untersuchungen unerlässlich, die auf der Grundlage einer standardisierten Erfassung aller durchgeführten Operationen und der Identifikation von komplikationsbehafteten Verläufen möglichst unverfälschte Ergebnisse liefern.

\subsubsection{Zur Definition der Begrifflichkeiten „Fehler“ und „Komplikation“}

An dieser Stelle muss eine kurze Schärfung der Begrifflichkeiten folgen, da im alltäglichen Sprachgebrauch der Verwendung der beiden Begriffe „Komplikation“ und „Fehler“ oft synonym und unkri- tisch erfolgt. Dem Wunsch, die Fachdiskussion mit einer einheitlichen Nomenklatur zu unterstützen wurde durch die Erarbeitung des „Glossars Patientensicherheit/Fehler in der Medizin“ durch das „Ärztliche Zentrum für Qualität in der Medizin“ Rechnung getragen. In diesem Glossar - erarbeitet von Experten aus Deutschland, der Schweiz und Österreich - wurden 2005 die im nationalen und internationalen Sprachraum gebräuchlichen Begriffe aus dem Bereich Patientensicherheit und Fehler in der Medizin zusammengeführt und entsprechend erläutert [146].

Komplikation: Nicht geplanter und/oder unerwarteter Verlauf, der die Heilung erschwert, beeinträchtigt oder vereitelt (auch unerwünschtes Ereignis). Eine Komplikation kann auch auftreten als schicksalhafter Krankheitsverlauf, etwa bei Verschlimmerung einer Erkrankung oder als Folge einer diagnostischen oder therapeutischen Maßnahme.

Fehler: Ein richtiges Vorhaben wird nicht wie geplant durchgeführt, oder dem Geschehen liegt ein falscher Plan zugrunde. Hier sind weitere Unterscheidungen zwischen Behandlungsfehlern, Zwischenfällen und Versagen zu treffen.

Hier soll und kann jedoch weder ein juristischer noch ein semantischer Exkurs folgen. Die prinzipielle Unterscheidung ist dennoch von Bedeutung, weil im Folgenden eben ausschließlich auf die „nicht geplanten“ und „unerwarteten“ Verläufe Bezug genommen werden soll. In der angelsächsischen Literatur werden zum Teil andere Begriffe und diese abweichend definiert, was wiederum zu differenten Kategorisierungen und damit zwangsläufig zu Unschärfe in der Diskussion führt. Die dortige Einteilung in und Definition von Operationsfolgen (engl. sequelae), Behandlungsmisserfolg (engl. failure to cure) und Komplikationen (engl. complications) sind daher ebenso Gegenstand kontroverser Diskussionen [147, 148], wie im deutschsprachigen Raum [144, 145].

Das Augenmerk muss aber gerade auf jene Verläufe gerichtet werden, bei denen der unerwünschte Verlauf unvorhersehbar eintritt. Beispielsweise die Fazialisparese, ohne Bohrarbeit oder Manipulation in topographischer Nähe zum Nerven, der Abfall der Knochenleitungshörschwelle ohne Arbeit an der Ossikelkette oder die Nachblutung trotz subtiler intraoperativer Blutstillung. Das technisch, wie ethisch und moralisch einwandfreie Handeln ist in diesem Moment der ärztlichen Tätigkeit immanent. Eben diese Verläufe sollen im Folgenden unter dem Begriff der Komplikationen zusammengefasst und betratet werden.

\subsubsection{Spezifische Komplikationen nach Ohroperationen}

Nicht alle möglichen, nach einer Mittelohroperation auftretenden Komplikationen sind zur spezifischen Qualitätsbewertung geeignet. Zweifelsohne lässt das gehäufte Auftreten von postoperativen Tiefenbeinvenenthrombosen eine Aussage über die Qualität des perioperativen Managements zu. Dennoch stehen sie nicht in einem direkten Zusammenhang mit der spezifischen chirurgischen Maßnahme einer Mittelohroperation. Anderseits sind Nachblutungen und Wundinfektionen nicht Ohroperationsspezifisch aber als lokale, im zeitlichen und ursächlichen Zusammenhang zur Operation auftretende unerwünschte Verläufe geeignet, das spezifische Behandlungsergebnis zu bewerten.

Ausgenommen von der Komplikationsbetrachtung sind zudem alle Rezidive, also bspw. die erneute Perforation, die erneute Retraktion bzw. das Cholesteatom-Rezidiv und dergleichen. Diese 
stellen eigene Parameter der Qualitätsbewertung, eher im Sinne des genannten Behandlungsmisserfolgs (engl. failure to cure) dar und sind an entsprechender Stelle abgehandelt worden.

\subsubsection{Retrospektive Komplikationsbetrachtung und prospek- tive Komplikationserfassung}

Die A Abb. 5 fasst wichtige Komplikationen zusammen, die nach mittelohrchirurgischen Eingriffen auftreten können. Eine feinere Untergliederung in frühe ( $<48$ Stunden nach Operation) und im späteren Verlauf (> 48 Stunden nach Operation) auftretende Komplikationen ist zudem sinnvoll.

Wie bereits erwähnt, ist die prospektive Studienlage zu diesem Thema nicht existent. Es konnten bisher keine publizierten Daten gefunden werden, die mittels einer prospektiven, strukturierten Registrierung einer Grundgesamtheit an Operationen und der darin zu verzeichnenden Häufigkeitsverteilung von Komplikationen generiert wurden. Folglich beziehen sich Häufigkeitsangaben und -verteilungen immer aus der retrospektiven Analyse von Krankenakten. Linder und Lin fassen die Problematik und Aussagekraft treffend zusammen: „Zudem genügt es nicht, lediglich die (...) vordefinierten Op.-Checklisten auszuwerten. Da kaum jemand die Zeit aufbringt, alle Op.-Berichte sorgfältig zu lesen (und aus dem fremden Text auch noch das Wichtigste zu erfassen), verkommen viele Langzeitstudien mit großen Fallzahlen zu wenig aussagenden Allgemeinplätzen“ [145].

Aufgrund dieser und weiterer Limitationen werden in der eigenen Abteilung (HNO-Universitätsklinik Dresden) nunmehr alle Ohroperationen routinemäßig registriert. Dabei erfolgt die Kennzeichnung aller komplikationsbehafteten bzw. nicht erwünschten Verläufe nach einem einheitlichen Schema der Parametererfassung. Die Auswahl derselben war das Ergebnis einer ersten retrospektiven Analyse, bei der die Komplikationen in Major- und Minor-Komplikationen eingeteilt wurden ( $>$ Abb. 5). Dabei wurden 377 Mittelohroperationen über einen 12-Monats-Zeitraum ausgewertet und hinsichtlich des Auftretens von Komplikationen in den ersten 6 Wochen nach Operation untersucht. Weiterhin wurden in dieser Studie Risikofaktoren erfasst und deren Korrelation mit dem Auftreten von Major- und Minor-Komplikationen untersucht. Diese Risikofaktoren waren der allgemeinchirurgischen Literatur entlehnt [149]. Hierbei konnte gezeigt werden, dass die aus der Allgemein- chirurgie bekannten Risikofaktoren in der Ohrchirurgie eine nachgeordnete Rolle spielen. Für die definierten Major-Komplikationen konnte generell kein statistischer Nachweis von Risikofaktoren identifiziert werden. Lediglich die arterielle Hypertonie stellt ein Risiko für postoperative Knochenleitungsabfälle dar $(R R=2,2$; $p=0,041)$.

-Tab. 1 zeigt eine Zusammenfassung des Auftretens, der Häufigkeit und dem Verlauf einzelner Komplikationen, nach der erwähnten prospektiven Erfassung über einen Zeitraum von neun Monaten (HNO-Universitätsklinikum Dresden). Die Kombination mit der Verlaufsbeurteilung rückt die Daten der reinen Eintrittshäufigkeit in ein differenziertes Licht. So mussten beispielsweise 32/419 (11\%) Patienten mit einem Knochenleitungsabfall (definiert als Abfall in 3 Frequenzen $>15 \mathrm{~dB}$, bzw. 2 Frequenzen $>20 \mathrm{~dB}$ ) in der unmittelbar postoperativen Phase identifiziert werden, davon haben sich jedoch bis zum Zeitpunkt der Analyse $n=21 / 419$ vollständig bzw. teilweise regredient gezeigt. Bei $n=11 / 419$ (2,6\%) blieb der KL-Abfall bis zum Analysezeitpunkt unverändert bestehen. Eine Ertaubung war nicht zu verzeichnen. Ähnlich verhält es sich mit der Affektion des N. fazialis. Alle drei aufgetretenen Affektionen haben sich im selben Beobachtungszeitraum von 9 Monaten vollständig oder bereits teilweise zurückgebildet. Aufgrund der Beobachtungsfortführung ist mit einer weiteren Regredienz der noch teilweise vorhandenen Paresen zu rechnen.

Die Aufteilung in Früh- (<48h post-OP) und Spätkomplikationen ( $>48$ h post-OP) macht zudem deutlich, dass ein Teil der insgesamt zu beobachtenden unerwünschten Verläufe erst nach der Entlassung auftritt. Weitere $n=14 / 419$ (3,3\%) Knochenleitungsabfälle waren zu beklagen, von denen wiederum $n=8 / 419$ vollständig, bzw. teilweise regredient und $n=6 / 419(1,4 \%)$ bis zum Ende des Analysezeitraums bestehen blieben. Somit sind von den 419 durchgeführten Mittelohroperationen in diesem Kollektiv insgesamt $n=46 / 419$ (10,9\%) postoperative Knochenleitungsaffektionen aufgetreten, von denen sich $17 / 419$ (4,0\%) als persistent erwiesen. Auch unter allen registrierten Fällen war keine Ertaubung zu verzeichnen.

Somit zeigt die routinemäßige, prospektive und strukturierte Komplikationserfassung erfreulicherweise, dass Major-Komplikationen insgesamt selten sind und sich der Großteil einmal beobachteter unerwünschter Verläufe wieder zurückbildet. Im Vergleich mit den wenigen publizierten retrospektiven Daten kann festge-

\section{Komplikationen}

Major-Komplikationen
I Knochenleitungsabfall
- (in 3 Frequenzen $\geq 15 \mathrm{~dB}$ oder
in 2 Frequenzen $\geq 20 \mathrm{~dB}$ )
I Fazialisparese
I Schwindel (mit Reiz-/Ausfallnystagmen)
I Tinnitus (mit Knochenleitungsaffektion)
I Wundheilungsstörung und - infektion mit
Notwendigkeit einer operativen Revision
I Liquorrhoe
I Intrakranielle Komplikationen, Meningitis

Minor-Komplikationen

I Schwindel ohne Nystagmus

I Wundheilungsstörung/- infektion ohne

notwendige Revision

I Tinnitus ohne Knochenleitungsaffektion

- Abb 5 Darstellung der definierten Major- und Minor-Komplikationen 
> Tab. 1 Komplikationen nach Mittelohroperationen (Universitätsklinikum Dresden). Unmittelbare Komplikationen (0-48h post OP, bzw. innerhalb des stationären Aufenthalts).

\begin{tabular}{|c|c|c|c|c|c|c|c|c|}
\hline & \multicolumn{2}{|c|}{$\begin{array}{l}\text { prozentualer Anteil an allen } \\
\text { Mittelohroperationen ( } n= \\
419) 09 / 2018 \text { bis } 05 / 2019 \\
\text { ( } 9 \text { Monate) (Universitätsklini- } \\
\text { kum Dresden) }\end{array}$} & \multicolumn{2}{|c|}{ Vollständig regredient } & \multicolumn{2}{|c|}{ Teilweise regredient } & \multicolumn{2}{|c|}{ Nicht regredient } \\
\hline & $n=$ & $\%$ & $\mathbf{n}=$ & $\%$ & $n=$ & $\%$ & $n=$ & $\%$ \\
\hline major & 46 & $11 \%$ & 24 & 5,7 & 10 & $2,4 \%$ & 12 & $2,9 \%$ \\
\hline Knochenleitungsabfall & $32 *$ & $7,6 \% *$ & 14 & $3,3 \%$ & 7 & $1,7 \%$ & 11 & $2,6 \%$ \\
\hline $\begin{array}{l}\text { Schwindel mit Reiz-/Ausfallnystagmen } \\
\text { oder SPN }\end{array}$ & 11 & $2,6 \%$ & 9 & $2,1 \%$ & 1 & $0,2 \%$ & 1 & $0,2 \%$ \\
\hline Fazialisparese & 3 & $0,7 \%$ & 1 & $0,2 \%$ & 2 & $0,5 \%$ & 0 & \\
\hline minor & 18 & $4,3 \%$ & 17 & $4,1 \%$ & 1 & $0,2 \%$ & 0 & \\
\hline Hämatom (retroaurikulär) & 7 & $1,7 \%$ & 7 & $1,7 \%$ & 0 & & 0 & \\
\hline Tinnitus & 6 & $1,4 \%$ & 5 & $1,2 \%$ & 1 & $0,2 \%$ & 0 & \\
\hline Nachblutung & 1 & $0,2 \%$ & 1 & $0,2 \%$ & 0 & & 0 & \\
\hline Schmeckstörung & 0 & & 0 & & 0 & & 0 & \\
\hline Schwindel ohne Nystagmen & 4 & $1 \%$ & 4 & $1 \%$ & 0 & & 0 & \\
\hline \multicolumn{9}{|c|}{ später eingetretene Komplikationen ( $>48 \mathrm{~h}$ post OP, bzw. nach dem stationärem Aufenthalt) } \\
\hline & $n=$ & $\%$ & $\mathbf{n}=$ & $\%$ & $\mathbf{n}=$ & $\%$ & $\mathbf{n}=$ & $\%$ \\
\hline major & 22 & $5,3 \%$ & 13 & $3,1 \%$ & 1 & $0,2 \%$ & 8 & $1,9 \%$ \\
\hline Knochenleitungsabfall & $14^{*}$ & $3,3 \% *$ & 7 & $1,7 \%$ & 1 & $0,2 \%$ & 6 & $1,4 \%$ \\
\hline Tinnitus & 4 & $1,0 \%$ & 3 & $0,7 \%$ & 0 & & 1 & $0,2 \%$ \\
\hline Fazialisparese & 3 & $0,7 \%$ & 3 & $0,7 \%$ & 0 & & 0 & \\
\hline Surditas & 1 & $0,2 \%$ & 0 & & 0 & & 1 & $0,2 \%$ \\
\hline minor & 57 & $13,6 \%$ & 51 & $12,2 \%$ & 2 & $0,5 \%$ & 3 & $0,7 \%$ \\
\hline Stenose & 25 & $5,9 \%$ & 22 & $5,2 \%$ & 0 & & 3 & $0,7 \%$ \\
\hline $\begin{array}{l}\text { Wundinfektion ohne notwendige } \\
\text { Revision }\end{array}$ & 17 & $4,1 \%$ & 15 & $3,6 \%$ & 2 & $0,5 \%$ & 0 & \\
\hline Dehiszenz & 5 & $1,2 \%$ & 5 & $1,2 \%$ & 0 & & 0 & \\
\hline Otorrhoe & 7 & $1,7 \%$ & 6 & $1,4 \%$ & 0 & & 0 & \\
\hline Taubheitsgefühle (Ohrmuschel, Zunge) & 1 & $0,2 \%$ & 1 & $0,2 \%$ & 0 & & 0 & \\
\hline Schwindel ohne Nystagmen & 1 & $0,2 \%$ & 1 & $0,2 \%$ & 0 & & 0 & \\
\hline $\begin{array}{l}\text { Wundinfektion oder -heilungsstörung } \\
\text { mit Notwendigkeit operative Revision }\end{array}$ & 1 & $0,2 \%$ & 1 & $0,2 \%$ & 0 & & 0 & \\
\hline Gesamt & 143 & $34 \%$ & 105 & $25 \%$ & 13 & $3,1 \%$ & 22 & $5,3 \%$ \\
\hline
\end{tabular}

*Definition eines Knochenleitungsabfalles: 3 Frequenzen >15dB, bzw. 2 Frequenzen > 20dB Abfall.

stellt werden, dass ähnlich detaillierte Aufstellungen bisher fehlen. - Tab. 2 zeigt, dass die Parameterauswahl im Vergleich gering ausfällt. Hinsichtlich der Knochenleitungsabfälle werden von Kazikdas et al. $n=18 / 51$ (35\%) beschrieben [150]. Von Phillips et al. werden retrospektive Daten zu Fazialisparesen, vestibulären Affektionen und Tinnitus, sowie Wundheilungs- und Schmeckstörungen geliefert. Die hier zu beobachtenden Differenzen sind am ehesten durch das retrospektive Studiendesign zu erklären. Die hierbei bekannten Limitationen kommen auch in den eigenen Auswertungen voll zum Tragen und unterstreichen die Notwendigkeit einer dauerhaf- ten, in die klinische Routine implementierten Registrierung und Erfassung von unerwünschten Verläufen. Nur so können valide Qualitätsbewertungen erfolgen, die im besten Fall als sich selbst generierende Kenngrößen innerhalb einer Abteilung zu jedem beliebigen Zeitpunkt heranziehen lassen.

Neben der bereits eingangs erwähnten sich weiter entwickelnden Fehlerkultur können abteilungs- und klinik-interne prospektive Erfassungen von Komplikationen daher enorm zu einer direkten Steigerung der Dokumentationsqualität und indirekt zu einer Verbesserung der Behandlungsqualität beitragen. 


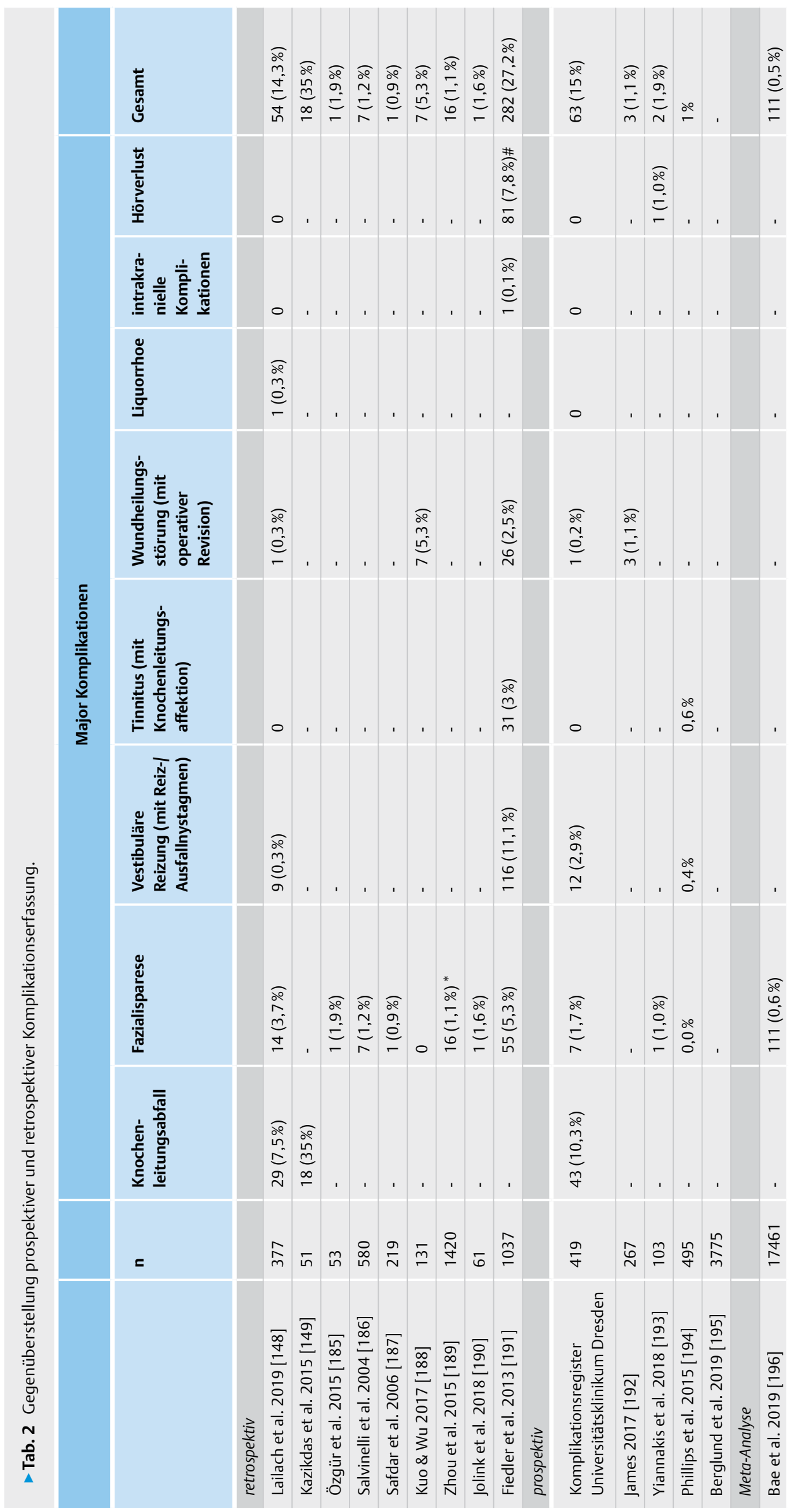




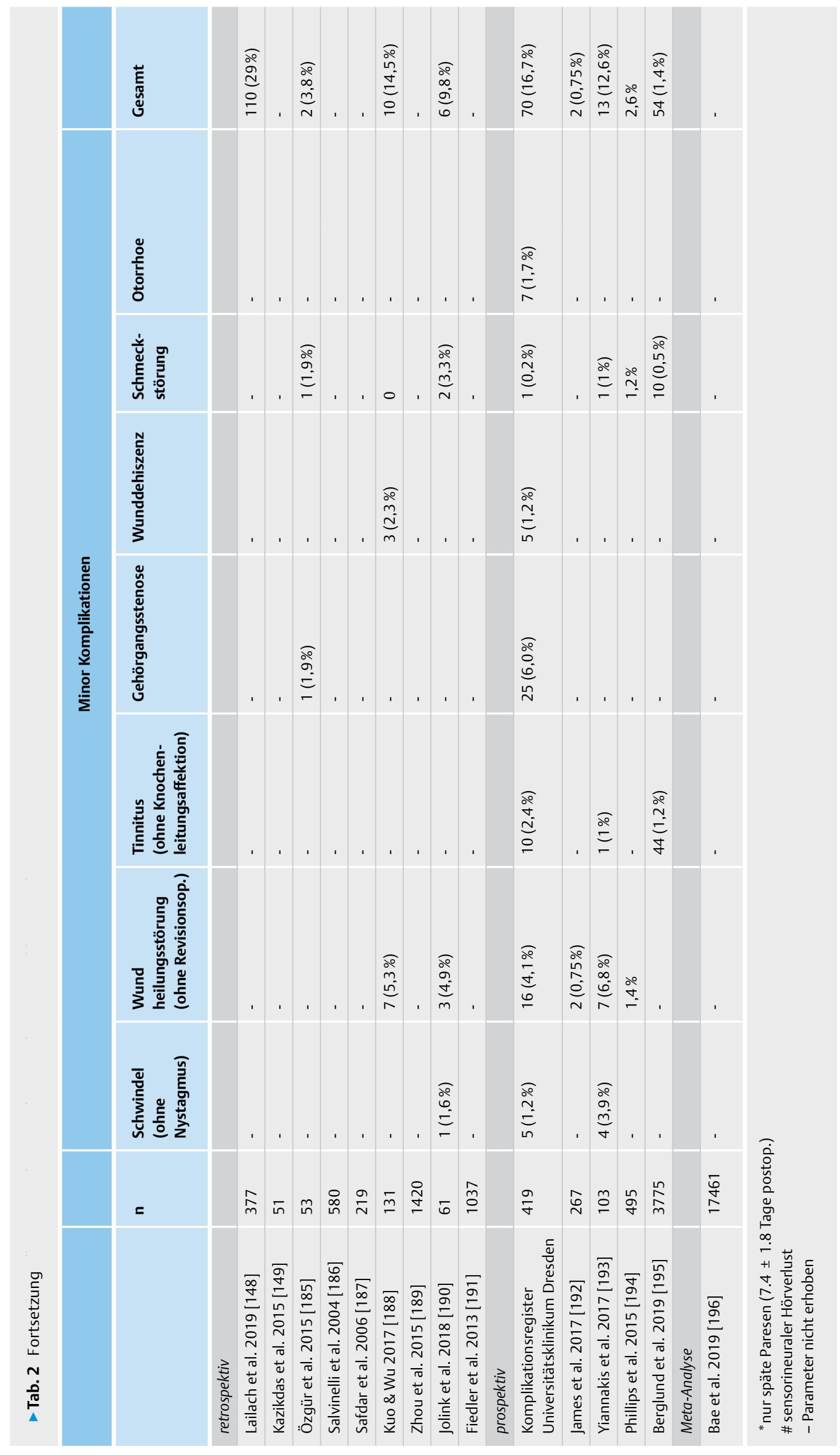




\section{Prozess- und Strukturqualität}

In dieser Kategorie können die Bereithaltung und Nutzung von Berichts- und Dokumentationssystemen zusammengefasst werden.

\subsection{Dokumentationsqualität}

Berichts- und Dokumentationsqualität sind essentielle Eckpfeiler in der klinischen Versorgung und der wissenschaftlichen Bewertung von klinischen Ergebnissen. Daher entscheidet die Güte, mit der Indikatoren der Ergebnisqualität beschrieben werden, direkt über die Belastbarkeit von formulierten Aussagen. Da in der klinischen Forschung eine Vielzahl von Faktoren das Ergebnis beeinflussen, kommt der Beschreibung der Ausgangs- und Beurteilungsbedingungen eine enorme Rolle zu. Alle im Vorfeld beschriebenen Qualitätsindikatoren der Mittelohrchirurgie sind in höchstem Maß von der Güte ihrer Erfassung, Beschreibung und Auswertung, sowie ihrer Interpretation abhängig.

\subsubsection{Unterschiede in Krankenversorgung und Forschung}

Für den einzelnen Patienten mögen nicht standardisierte Mess- und Dokumentationsbedingungen weniger ins Gewicht fallen, wenn systematische Einflussfaktoren konstant gehalten und „nur“ individuelle Verläufe, longitudinal betrachtet werden. Aber bereits der Wunsch eine eigene, überschaubare Patientenkohorte zu bewerten setzt eine detaillierte und damit standardisierte Charakterisierung voraus, um Aussagen über das Individuum hinweg treffen zu können. Für klinische Studien mit dem Anspruch Evidenz zu generieren müssen die beeinflussenden Hauptfaktoren klar definiert werden.

Für die rekonstruktive Mittelohrchirurgie lassen sich folgende Minimalkriterien zur Dokumentation festgehalten:

- Studienpopulation (Art und Ausmaß der behandelten Pathologie)

- Intervention (Art und Umfang der Behandlung/Operation)

- Ergebnisbeurteilung (Art, Umfang und Zeitpunkte der Erhebung)

Kurz gesagt, gelten hier die Grundsätze guten wissenschaftlichen Arbeitens, nach denen beschrieben wird „was“, „wie“ und „wann“ gemacht wird. Auch wenn die Medizin immer wieder allgemeinhin als „nicht exakte“ Wissenschaft bezeichnet wird, sollte diese Aussage auf der Berücksichtigung weit streuender individueller Abweichungen im biologischen System „Mensch“ beruhen, aber nicht auf einer Nachlässigkeit in der Erhebung und im Umgang mit Quelldaten und Messergebnissen. Die Anwendung grundlegender naturwissenschaftlicher Kenntnisse bei der Ergebnisbeschreibung darf nach Abschluss eines Hochschulstudiums unausgesprochen vorausgesetzt werden. Daher mag auf den ersten Blick die alltägliche Routinedokumentation in der Krankenversorgung von den Ansprüchen wissenschaftlichen Arbeitens abweichen, aber eben nur auf den ersten Blick. Auch hier müssen die o.g. Aspekte aus medikolegalen Gründen umgesetzt werden, in der Regel jedoch nicht immer so umfassend und strukturiert, wie es für eine wissenschaftliche Aufarbeitung der Ergebnisse nötig ist. Dies führt einerseits zu den bekannten Problemen retrospektiver Studien, bei denen im Nachgang nur noch mit den einst dokumentierten Daten gearbeitet werden kann. Alle in den Quelldokumenten nicht beschriebenen Aspekte müssen unberücksichtigt bleiben. Anderseits wurden allerorts zahlreiche Lis- ten mit Symptomen, Befunden und Prozeduren entworfen. Aufgrund der Uneinheitlichkeit in Nomenklatur, Umfang und Interpretation sind diese nur bedingt oder gar nicht miteinander vergleichbar.

Dies hat bereits sehr früh dazu geführt, Klassifikations- und Bewertungssysteme zu etablieren. In der rekonstruktiven Mittelohrchirurgie existiert seit 1956 die Klassifikation nach Wullstein [151], die neben der Beschreibung der Rekonstruktionsarten des Mittelohres auch das davon abhängig beobachtete Hörergebnis beinhaltete. Auch Bellucci beschrieb 1969 ein duales Bewertungssystem, welches das Risiko für eine erfolgreiche Tympanoplastik an dem Vorhandensein, bzw. der Neigung zu Mittelohrinfektionen misst [152]. Zahlreiche weitere sollten folgen.

Der Übergang von der Dokumentation in der klinischen Routine der Patientenversorgung und der Auswertung der Ergebnisse zu wissenschaftlichen Zwecken sind fließend. Allgemein verfolgen sie aber immer die drei Ziele: (1) die Beurteilung einer Operationsmethode oder einer Rekonstruktiontechnik (bzw. einer Prothese), (2) die Vergleichbarkeit der Ergebnisse mit anderen Fallserien und Studien und sie dient (3) dem Zweck der Erfolgsprognose (für Patient und Arzt) $[117,122]$.

\subsubsection{Beschreibungs- und Dokumentationsstandards}

Die Klassifikationen nach Wullstein und Bellucci wurden bereits erwähnt. In den Folgejahren kamen zahlreiche weitere hinzu mit unterschiedlicher Durchdringungskraft und Präsenz in der ohrchirurgischen Literatur. Die bekannteren sind die „SPITE-Kriterien“ von Black [153], die Austin Klassifikation, meist in der Modifikation nach Kartush [154], aus der sich der „Middle Ear Risk Index“, MER-Index, kurz MERI ableitet $[155,156]$. Als einziger statistisch begründeter Index ist der OOPS-Index (engl. Ossiculoplasty Outcome Parameter Staging Index) zu nennen [157]. Aber auch für spezielle Aspekte der Trommelfellrekonstruktion mit Knorpel [158] und die endoskopische Ohr-Chirurgie [159] stehen Klassifikationssysteme zur Verfügung.

Den existierenden Klassifikationen für das Cholesteatom wurde unlängst eine Übersichtsarbeit gewidmet [160] auf die hier inhaltlich im Einzelnen nicht eingegangen werden soll. Die aktuelle Europäische Klassifikation der „European Acadamy of Otology and Neurotology“ zusammen mit der Japanischen Otologischen Gesellschaft (EAONO/JOS) stammt aus dem Jahr 2017 [161]. Sie umfasst die Definition, die Klassifikation und das Staging (Bewertung) des Cholesteatoms. Erarbeitung und Beschluss erfolgten vor, während und nach der 10. Internationalen „Cholesteatom und MittelohrChirurgie“ Konferenz im Jahr 2016 (Chole2016). Abschließend wurde die Definition von $89 \%$, die Klassifikation von $98 \%$ und das Staging von $75 \%$ der internationalen Delegierten angenommen. Die Bildung einer internationalen „Otology Outcome Group“ (IOOG) wurde zur Erarbeitung eines allgemeinen, minimalen Berichtsstandards für die Verwendung der internationalen otologischen Gemeinschaft in Aussicht gestellt.

Die jüngste Publikation, stellt die von der eben genannten IOOG, zur Kategorisierung der Tympanomastoid-Chirurgie dar [109]. In einem ähnlichen Verfahren wurde darin ein Konsens zur Beschreibung der Mittelohrchirurgie erarbeitet. Die Abkürzung SAMEO-ATO steht darin für die Bewertungskategorien. Ein großer Vorteil könnte die simultane Illustration der definierten Merkmalsausprägungen sein, da hierdurch der Interpretationsspielraum deutlich ein- 
geschränkt wird. Auch dieser Konsens wurde von einer Gruppe internationaler Delegierter abschließend mit einer deutlichen Mehrheit verabschiedet (95\% (20/21) bis $100 \%$ (21/21) je nach Merkmal).

Diese beiden im Delphi-Verfahren gestalteten, sehr aufwendigen Prozesse zeigen gut, wie komplex und schwer die Konsensfindung im internationalen Kontext ist. Die nächsten Jahre werden zeigen, ob sich die propagierten Konsense durchsetzen. Angesichts der großen Zahl existierender, vorgeschlagener und beschriebener Systeme, scheint die Einigung auf einen internationalen Standard eine nahezu wohltuende Erleichterung in Aussicht zu stellen. Der Erfolg des Projekts liegt dabei aber in der Hand der internationalen otologischen Gemeinschaft selbst, da nur sie die vereinbarten Konsense konsequent anwenden und ihnen damit zum Erfolg verhelfen kann. In der logischen Konsequenz bedeutet die Anerkennung der neuen Klassifikationen und Kategorisierungen entweder die zusätzliche oder alleinige Codierung der Prozeduren, unabhängig von eventuell bereits lokal etablierten Dokumentationsschemata. Gerade hier kann auf Arbeitsebene Widerstand antizipiert werden, da unter Umständen arbeitsintensive oder technisch nicht realisierbare „Umcodierungen“ von einer Klassifikation in die neue stattfinden müssten. Darüber hinaus sind neben den technischen Herausforderungen auch persönliche Befindlichkeiten zu befürchten, wenn eine internationale „Otology Outcome Group“ bzw. ein Lenkungsausschuss derselben einen Konsens propagiert, in dessen Entstehungsprozess nie jeder gehört und nie alle Meinungen berücksichtigt werden können. Daher sind die Verabschiedungen der beiden Konsense auch ein Appell an die internationale otologische Gemeinschaft persönliche Sichtweisen hinten an und in den Dienst der Sache zu stellen.

\subsubsection{Anwendung von Klassifikations-Systemen und Berichts- standards}

Übertragen auf den scheinbar kleinen Teilaspekt des Ergebnisberichtsstandards bei Operationen zur Therapie der Schalleitungsschwerhörigkeit, können die Empfehlungen der amerikanischen Fachgesellschaft AAO-HNS aus dem Jahr 1995 als Minimalkriterienkatalog angesehen werden [123]. Ähnlich wie die beiden o.g. Kategorisierungssysteme zum Cholesteaom und der Tympanomastoid-Chirurgie wurde hier der Versuch unternommen, einen Standard für die Outcome-Beschreibung festzulegen. Im Gegensatz zu den beiden Konsensprozessen stellte dieser einen Vorschlag der AAO-HNS dar.

Akzeptanz und Durchdringung der Empfehlungen legte eine kritische Analyse der Literatur im Zeitraum von 2005 bis 2015 dar, die zum Thema „Hörergebnisse nach Mittelohroperationen“ durchgeführt wurde [162]. Diese zeigte, dass bereits bei der Beschreibung der methodologischen Rahmenbedingungen der Studien grundlegende Mängel bestehen. Die Anwendung mathematischer und Test-statistischer Grundlagen bei der Angabe von Hörergebnissen und ihrer Veränderungen in Form von Mittelwerten mit Standardabweichungen darf in einer wissenschaftlichen Disziplin eigentlich vorausgesetzt werden. Dennoch waren in der Untersuchung von 169 Publikationen (allesamt in begutachteten, „peer-review“ Zeitschriften) der postoperative ABG nur in $56 \%$ in dieser Form angegeben. Weiterhin fehlten in $17 \%$ Aussagen zu den angewendeten statistischen Methoden. Die von der AAO-HNS empfohlenen Prüffrequenzen wurden in weniger als der Hälfte (46\%) angewendet und Angaben hierüber fehlten in 15\% vollständig. Streng genommen können aus den Ergebnissen dieser Studien aufgrund dieser Mängel keine Aussagen abgeleitet werden. Betrachtet man weiter die Anwendung der 10 Kriterien des AAO-HNS 1995 Standards, so wurden diese in keiner Publikation vollständig korrekt angewendet und in 5\% (9/169) überhaupt nicht. Eine Korrelation korrekt angewendeter Kriterien mit dem impact factor der Fachzeitschrift bestand nicht $(r=0,008 ; p=0,3)$.

Eine weitere Untersuchung zur Anwendung und Beschreibung der Sprachaudiometrie in Studien zu hörverbessernden Mittelohroperationen, implantierbaren Hörsystemen und Therapie von Kleinhirnbrückenwinkel-Tumoren (zwischen 2012 und 2016) bestätigt die Grundzüge des vorliegenden Problems (Morgenstern und Lailach et al., 2019 in review): in 20 \% (56/279) fehlten Angaben zu statischen Testverfahren, in $11 \%$ (32/279) bzw. in $13 \%$ (12/279) Aussagen über die Prospektivität bzw. das Studiendesign. Insbesondere die Angabe des messtechnisch so sensiblen Parameters der „Sprachverständlichkeit“ war alarmierend lückenhaft. Zwar verwendeten $90 \%$ (252/279) der Studien diesen Parameter, aber in $60 \%$ (167/279) fehlten Angaben über den Darbietungsschalldruck und die Messbedingungen, was die Aussagen nahezu vollkommen entkräftet. Des Weiteren ist hier exemplarisch der interessante Effekt einer „fortgesetzten Unschärfe“ zu beobachten. In $45 \%$ der Studien zur Behandlung des Vestibularisschwannoms bezog man sich bei der Beschreibung der audiometrischen Funktionstestung auf eine Publikation von Gardner und Robertson aus dem Jahr 1988 [163]. Allerdings wird in der zitierten Originalarbeit gar kein audiologisches Messverfahren beschrieben, sondern eine Klassifikation mit den Kategorien „alltagstaugliches und nicht-alltagstaugliches Gehör“. Die Autoren empfehlen die Anwendung ihrer Klassifikation als zusätzliches Mittel neben entsprechenden audiologischen Testverfahren. Neue Berichtsstandards können erst mit einer gewissen zeitlichen Verzögerung in der Literatur berücksichtigt und umgesetzt werden [164]. Zusammenfassend muss man an dieser Stelle festhalten, dass es nicht am Fehlen von Berichts- und Dokumentationsstandards fehlt, sondern diese nicht oder nur teilweise angewendet werden.

Interessant ist an dieser Stelle die Frage nach den Gründen und Konsequenzen aus den Erkenntnissen. Ursächlich können die Praktikabilität und/oder die Güte eines Berichtsstandards selbst sein. Einige Autoren mögen einen Standard in Teilen kritisch betrachten oder gar inhaltlich komplett ablehnen. Dies erklärt jedoch nicht die Mängel bei der Anwendung des methodischen „Handwerkszeugs“. Daher muss hier auch die unangenehme Frage nach der Sinnhaftigkeit und dem Nutzen des peer review-Verfahrens gestellt werden. Es zeigt sich nämlich nicht nur, dass autoren-seitig gründlicher gearbeitet werden muss, sondern auch, dass das Begutachtungsverfahren teilweise nicht vollumfänglich greift [162].

\subsection{Erfassungs- und Dokumentationssysteme}

Historisch gewachsen stellt die Mitteohrchirurgie keinen Sonderfall dar, wenn es um die wissenschaftliche Begründung für spezifische Therapie- oder Operationsstrategien geht. Die Entscheidungsgrundlage ist oftmals empirisch und gegründet auf die klinischen Beobachtungen und Erfahrungen, die seit den Anfängen der Mittelohrchirurgie bis heute gesammelt wurden $[165,166]$. Diesem 
Umstand wäre durch prospektive, kontrollierte und randomisierte Studien zu begegnen, die an den Grundsätzen guter wissenschaftlicher und klinischer Praxis durchgeführt werden und eine repräsentative Anzahl an Patienten aufweisen. Gründe dafür, dass dies auch in den letzten Jahren schwer zu realisieren war, sind vielfältig:

1. Bewertungs- und Klassifikationssysteme: Die existierenden Bewertungs- und Klassifikationssysteme, bzw. publizierte Berichtsstandards [165, 167-169] werden nur teilweise oder gar nicht verwendet $[133,170]$. Da retrospektiv im Freitext gehaltene Anamnesen und Operationsberichte nicht valide in standardisierte Bewertungsinstrumente überführt werden können, ist die Beschreibung einer homogenen Studienpopulation schwer bis nicht möglich. Dies führt unweigerlich dazu, dass auf diese Weise generierte Aussagen den Charakter von individuellen Ergebnisberichten haben.

2. Nomenklatur: ist sehr unterschiedlich und bietet daher viel Raum für Interpretationen, die einer eindeutigen Befund- und Vorgehensbeschreibung entgegenwirken.

3. Vielzahl an Einflussfaktoren: Die individuelle Mittelohrpathologie wird von einer Vielzahl zusätzlicher Faktoren beeinflusst, so dass die statistisch zu fordernde Kontrolle einzelner oder mehrerer Faktoren in Patientenpopulationen nahezu unmöglich ist. Eine durchaus respektable Anzahl von Patienten kann somit schnell auf eine einstellige Populationsgröße schrumpfen, was statistische Auswertungen unmöglich macht.

4. Einflussfaktor Operateur: Zusätzlich zur individuellen Pathologie, bestehen große Unterschiede in Operationsstrategie und Expertise zwischen verschiedenen Standorten. Dies wird durch die Anzahl der beteiligten Operateure einer Abteilung multipliziert. Dadurch sind bereits innerhalb einer Abteilung vergleichende Ergebnisbewertungen bei mehreren Operateuren bereits erschwert.

5. Retrospektives Studiendesign: Das retrospektive Design der überwiegenden Studien bedingt eine Beeinträchtigung der Datenqualität (u.a. durch die vorgenannten Punkte) und stellt ein großes Risiko für design-bedingten Bias dar.

6. Zusätzlicher Dokumentationsaufwand: In den wenigsten Zentren werden in der Routine die wichtigsten Einflussfaktoren und Prädiktoren für das Behandlungsergebnis systematisch erfasst. Die Pflege von Datensätzen zur statistischen Auswertung für wissenschaftliche Untersuchungen stellt eine immense Zusatzbelastung für den ohnehin mit Dokumentation überfrachteten Klinikalltag dar.

7. Datenbanksysteme: Der „Würzburger Ohrbogen“ [171] wurde in den Neunzigerjahren entwickelt und stellt sicher eines der ersten, brauchbaren computergestützten Instrumente zur standardisierten Erfassung von Ohroperationen dar. Auch zuvor wurden in Deutschland bereits standardisiert Daten zu Ohroperationen gesammelt und erfasst $[172,173]$. Damals zeitgemäß mit Lochkartensystemen und den Möglichkeiten der computerisierten Informationstechnologie [174]. Mit dem „Würzburger Ohrbogen“ wurden seinerzeit über 10000 Ohroperationen größtenteils inklusive Audiogramme und Nachsorgeuntersuchungen in einem MS DOS basierten Datenbanksystem eines einzigen Standorts erfasst. Zur Zeit seiner Entstehung war dies eine richtungsweisende Entwick- lung, die leider durch die fortschreitende Computertechnologie „überholt“ wurde. Eine Anpassung an zeitgemäße Betriebssysteme blieb aus, was die Limitationen hauseigener, nicht-kommerzieller Lösungen verdeutlicht.

Mittlerweile existieren frei zugängliche Datenbanksysteme, die internet-basiert die Eingabe und Auswertung von Daten erlauben. Auch die standortübergreifende Auswertung von Ergebnissen ist somit möglich, was die wichtigste Funktion im Sinne der Evidenzorientierung darstellt. Leider werden diese Datenbanken zu wenig genutzt [175]. Neben dem o.g. zusätzlichen Zeitaufwand bestehen oft Ressentiments zur „Ab-“ oder „Weitergabe“ von persönlichen Datensätzen. Dies mag in einer befürchteten Rückverfolgbarkeit, einer generellen Angst des Missbrauchs von anonymisierten Patientendaten an Dritte oder anderen Gründen liegen.

Die genannten Punkte verdeutlichen die Schwierigkeiten, denen der Qualitätsanspruch bei der Generierung wissenschaftlich fundierter Ergebnisbewertungen entgegensteht. Andererseits kann diesem Problem nur durch eine routinemäßige, systematische, und prospektive Erfassung bzw. Sammlung von Erkrankungs- und Therapiedaten begegnet werden. Somit kristallisiert sich im Kern die Verfügbarkeit und die Nutzung von Datenbanksystemen heraus und daher Überlegungen des Datenschutzes und des Bedien-, Zeitund Kostenaufwandes. Ein Mehr an Qualität ist gleichzusetzen mit einem Mehraufwand an Dokumentation, der in die Routine fest implementiert werden muss. Es erübrigt sich zu erwähnen, dass dieser Mehraufwand im Refinanzierungskonzept unseres Gesundheitssystems bisher nicht eingepreist ist. Nichtsdestotrotz kann auch zukünftig nur so ein höheres Maß an Evidenz generiert werden.

Diesen Zielen folgend sind bereits verschiedene standortübergreifende Datenbanksysteme entwickelt worden, die unterschiedlich weite Verbreitung gefunden haben. Einige davon sollen vorgestellt werden.

\subsubsection{Common Otology Audit Database}

Im Jahr 2004 gegründet und 2005 als „International Otologic Database“ publiziert [176], stellt dieses Datenbank die erste internationale, Länder- und Standortübergreifende Datenbank für Mittelohrchirurgische Eingriffe dar. In der Pilotphase haben drei der Autoren jeweils 50 Datensätze für Otosklerose-Operationen eingepflegt. Aufgrund dieser Erfahrung wurde die Dateneingabe als benutzerfreundlich und schnell (ca. 2 Minuten/Datensatz) bewertet und laut der Autoren stimmten alle bisherigen Nutzer dieser Aussage zu [176]. Die Datenabfrage gliedert sich in 2 Kategorien. Die Basisdatenbank-Eingabe erfasst nur einige Kriterien und wertet folglich eingeschränkte Ergebnisparameter aus. Demgegenüber erlaubt die Eingabe über Kategorie 2 eine umfassende Analyse anhand ausgedehnter Parameterabfragen. Diese umfasst sowohl präoperative, anamnestische Angaben zur Pathologie und zu den Symptomen, intraoperative Befunde und durchgeführte Maßnahmen und erlaubt postoperative Nachbeobachtungen zu festen Zeitpunkten. Zudem können prä- und postoperative Reintonaudiogrammwerte der Luftund Knochenleitungshörschwellen eingetragen werden, sodass automatisch der ABG berechnet wird. Die Zuordnung erfolgt bezogen auf den anonymisierten Patienten über eine Eingabeidentifikations- 
- Tab. 3 Zusammenstellung von Datenbanksystemen und Registern zur Erfassung und Auswertung vom Mittelohr-Operationen.

\begin{tabular}{|c|c|c|c|c|c|}
\hline Name & Sprache & Jahr & $\begin{array}{l}\text { Beschrei- } \\
\text { bung }\end{array}$ & $\begin{array}{l}\text { Kommer- } \\
\text { ziell }\end{array}$ & Publikationen \\
\hline Common Otology Audit Database & Englisch & 2004 & {$[176]$} & nein & {$[175,186-189]$} \\
\hline Standardized Korean Ear Surgery Database & Koreanisch & 2005 & {$[190]$} & nein & [181] \\
\hline OtoDatabase & Dänisch & 2002 & [182] & nein & [182] \\
\hline Otology-Neurotology Database & Englisch & 2006 & [183] & ja & {$[183,188,191-194]$} \\
\hline OtoKir Database & Englisch & 2004 & [8] & nein & {$[8,195-196]$} \\
\hline ENTstatistics & Deutsch & 2004 & & ja & \\
\hline Swedish National Quality Registry & Schwedisch & 2004 & {$[184]$} & nein & {$[184,197-198]$} \\
\hline
\end{tabular}

nummer sowie auf den Chirurgen. Das System bietet jedem beitragenden Ohrchirurgen die Möglichkeit, seine Ergebnisse aufgeschlüsselt nach den Kategorien Stapeschirurgie, Myringoplastik, Ossikuloplastik und Cholesteatomoperation (Kinder und Erwachsene) zu beziehen und diese abgeglichen auf die Gesamtheit aller Einträge dargestellt zu bekommen (benchmark database). Exportierbar in gängige Tabellenkalkulations- und Statistikprogramme sind sie der wissenschaftlichen Bearbeitung zugänglich.

Zum Zeitpunkt der Publikation hatten sich 27 Otologen aus 12 Ländern, die „European Otology Database Project Group“, auf den standardisierten Datensatz zur Abfrage geeinigt, darunter auch deutsche Vertreter.

\subsubsection{Standardized Korean Ear Surgery Database}

Im Jahr 2001 hat die „Korean Otologic Society“ eine standardisierte Datenbank für Mittelohrchirurgische Eingriffe erstellt [177]. Dieses nationale Projekt beinhaltete die Standardisierung einer mittelohrchirurgischen Nomenklatur und Empfehlungen für die postoperative Ergebnisberichtsdarstellung. Bei der Konsensfindung waren 9 Ohrchirurgen von 7 Universitäten beteiligt. Dabei wurden nicht nur Standards für die Ergebnisbewertung festgelegt, sondern zudem eine einheitliche Nomenklatur definiert. Hierzu erfolgte die Orientierung an internationalen Vergleichsarbeiten, bspw. aus Japan [178], Europa [176], aber auch anderen Klassifikationen [166, 179, 180]. Wie auch bei den anderen Dokumentationssystemen wird hier die Herausforderung in der Datenbankpflege gesehen. Aufgrund der in Koreanisch gehaltenen Dokumentationslisten ist die Anwendung dieses Systems in internationalen Kontext limitiert.

Im Jahr 2012 wurde mithilfe der Datenbank eine Serie von 2312 Operationen eines einzelnen Chirurgen zwischen 1989 und 2009 zur Therapie der chronischen otitis media ausgewertet [181]. Da die Datenbank erst 2005 entwickelt wurde, sind die Daten nachträglich eingepflegt worden, was alle Einschränkungen der retrospektiven Datenüberführung mit sich bringt. Dennoch zeigt dieses Beispiel eindrücklich, welche Möglichkeiten die Verwendung von Datenbanksystemen bieten können. Im Ergebnis fanden die Autoren, dass die Funktion der Tuba auditiva, das Vorliegen eines Cholesteatoms und das Ausmaß der Ossikelzerstörung (hauptsächlich die des Stapes) das postoperative Ergebnis beeinflussen.

\subsubsection{Oto-Database}

Eine Gruppe niederländischer Autoren aus Rotterdam beschrieb 2002 eine hauseigene Datenbank, die zur internen Aufzeichnung und Auswertung von Ohroperationen verwendet wird [182]. Neben der detaillierten Beschreibung der Dokumentationslisten setzen sie sich intensiv und kritisch mit Nutzen und Aufwand der elektronischen Datenaufzeichnung auseinander. Im Durchschnitt war eine 2- bis 3-minütige Dokumentationszeit für die Eingabe der Operationsdaten nötig. Von den 1.009 Datensätzen zum Zeitpunkt der Publikation, war der OP-Berichtsbogen in $89 \%$ ausgefüllt. Diese Rate fiel für die ambulanten Folgevisiten drastisch auf $2 \%$ ab. Die Autoren heben insbesondere auf den Wert der Ergebnisbewertung für das individuelle Feedback an den Operateur und für die Abteilung ab. Es werden ausführlich strukturelle Daten diskutiert und bewertet. Weitere Veröffentlichungen unter Verwendung dieses Datenbanksystems konnten nicht gefunden werden.

\subsubsection{Otology-Neurotology Database}

Die Otology-Neurotology Database (ONDB) [183] wurde 2006 von Vincent R. und Kollegen als kommerziell zu erwerbendes SoftwarePaket vorgestellt, welches an der eigenen Klinik entwickelt wurde. Der Berichtsformat-Standard der AAO-HNS 1995 wurde hinterlegt und ein internationales wissenschaftliches Komitee zur Weiterentwicklung und Beratung gegründet. Die Anwendung sah zum Zeitpunkt der Vorstellung ausschließlich die Registrierung von otologischen Patienten und Befunden vor, sollte aber nicht nur auf neurootologische Erkrankungen und den gesamten HNO-Bereich ausgedehnt werden. Die Software sah bereits eine multizentrische Nutzung mit Daten-Pooling innerhalb einer Institution und deren Grenzen hinweg vor. Mit der Inauguration des Datenbanksystems wurde in gleicher Publikation die stattliche Zahl von 3050 Stapesoperationen im Zeitraum von 1991 bis 2004 ausgewertet.

\subsubsection{OtoKir Database}

Die OtoKir Datenbank wurde in Kopenhagen, Dänemark entwickelt [8]. Vergleichbar mit dem „Würzburger Ohrbogen“ handelt es sich um eine hauseigene Datenbank, mit der die Erfassung von Eingriffen und ergebnisspezifischen Einflussfaktoren möglich ist. Eine automatisierte Schnittstelle erlaubt den Import von Audiogrammdaten, wenn diese in elektronischer Form vorliegen. Die Autoren 
betonen eine hohe Nutzerfreundlichkeit, obgleich diese bei der kritischen Betrachtung Einschränkungen erfährt. Die Registrierung läuft in weiten Teilen parallel zum Krankenhausinformationssystem (KIS), die Operateure müssen die zusätzliche, teilweise doppelte Dokumentationsarbeit leisten und der Import von externen Quelldaten, bspw. Audiogrammen muss über Schnittstellen bewerkstelligt werden. Sehr positiv hervorzuheben ist, dass die Datenbank von der Dänischen Vereinigung der Ohrchirurgen landesweit eingesetzt und weiterentwickelt werden soll [8]. Zudem ist sie frei zugänglich auf Ebene des Microsoft Betriebssystems installierbar.

\subsubsection{Swedish National Quality Registry for Myringoplasty}

Das schwedische Myringoplastik Register wurde 1997 eingeführt [184]. Es handelt sich nicht um ein Datenbank-System im eigentlichen Sinn, da sich aber im Verlauf alle 33 schwedischen HNO-Kliniken beteiligt haben, soll es exemplarisch als nationale Qualitätsinitiative vorgestellt werden. In welcher Form die Datenerhebung erfolgte ist nicht explizit erwähnt. Nach der Etablierungsphase wurden zwischen 2002 und 2012 insgesamt 6334 Prozeduren registriert. Neben anamnestischen Daten wurden audiologische Daten (Reintonaudiogramm) und Informationen zur Operationstechnik aufgenommen. Die Menge an registrierten Daten wurde bewusst geringgehalten, um eine hohe Compliance bei der Dateneingabe zu erzielen, die an insgesamt 4 Zeitpunkten ( 1 präoperativ, 3 postoperativ) erfolgte. Die Daten hinsichtlich der GTR sind mit 89,5\% in Einklang mit den im Kapitel 3.1.1 dargestellten Daten.

Die Autoren sehen die Vorteile des Registers in der Möglichkeit des Datenaustauschs und -pooling zwischen den teilnehmenden Zentren und der Qualitätskontrolle. Daraus leiten sie den Vorteil eines nationalen Lernprozesses und eine stetige Verbesserung des schwedischen Gesundheitssystems ab.

\subsubsection{ENTstatistics}

Das Programm ENTstatistics der Firma Innoforce ist eine kommerzielle Datenbank zur Verwaltung von Ohr- und anderer HNO-Operationen. Über entsprechende Software-Schnittstellen ist die Kommunikation zum KIS und der Gerätesoftware der Audiometrie möglich. Auch Fragebögen von HRQOL Messinstrumenten und andere individuelle Fragelisten lassen sich integrieren. Zudem besteht prinzipiell die Möglichkeit des Datenpooling von verschiedenen Anwendern und eine gezielte Parameterabfrage. Aufgrund der kommerziellen Nutzung sind Datenbankpflege und Instandhaltung sichergestellt. Publikationen, die sich konkrete auf die Anwendung des Systems bei Mittelohroperationen beziehen, sind bisher nicht bekannt. Über eine Nutzung im Bereich der Cochlear Implantation ist aus Heidelberg berichtet worden [185].

Zum Teil sind aus der Nutzung der o.g. Datenbanksysteme bereits Ergebnisse für eine Reihe von Publikationen extrahiert und analysiert worden ( $\triangleright$ Tab. 3) . Dabei werden die Vorteile schnell deutlich: die konsequente Nutzung führt zu großen Fallzahlen, die auch nach Filterung für bestimmte Parameterausprägungen noch respektable Populationsgrößen für statistische Auswertungen liefern. Besteht zudem die Möglichkeit zum nutzerübergreifenden Datenpooling, steht detaillierten Analysen in Form von prospektiven, multizentrischen Studien nichts im Wege. Allerdings zeigt sich auch hier, dass die Vielzahl von bereits existierenden lokalen und/ oder nationalen, z. T. selbsterstellten Systemen diesem Ziel nicht zuträglich ist. Demgegenüber sind die Anschaffung, Anbindung an die Krankenhausinformationsstruktur, Pflege und Instandhaltung der kommerziellen Lösungen entsprechend hochpreisig und nicht ohne weiteres in ein begrenztes Investitionsbudget integrierbar.

Zusammenfasend können die Anforderungen an ein Dokumentationssystem die folgenden Anforderungen gestellt werden:

- Kostengünstig, bzw. angemessene Kosten-Nutzen Relation

- Unkomplizierter Datentransfer zum Krankenhausinformationssystem

- Unkomplizierter Datentransfer von Quelldaten (Audiometrie, HRQOL, parametrierte KIS-Formulare)

- Professionelle Pflege und Instandhaltung der Datenbank

- Möglichkeit zum individuellen Datenabruf und Parameterübergreifender Auswertung

- Informationsstruktur zur Bearbeitung und Auswertung von standortübergreifenden wissenschaftlichen Fragestellungen unter Einhaltung datenschutzrechtlicher und ethischer Aspekte.

- Problemlose Integration neuer Klassifikationssysteme mit der Möglichkeit einer nachträglichen Umcodierung von bereits vorhandenen Datenbankeinträgen

\section{FAZIT}

Die Feststellung von Aristoteles scheint die Ausführungen in geeigneter Weise zusammenzufassen: „Wir sind das was wir wiederholt tun. Vorzüglichkeit ist daher keine Handlung, sondern eine Gewohnheit.“ [Aristoteles (384-322 v. Chr.)]. Der Qualitätsbegriff kommt in der englischen Übersetzung vor: „Quality is not an act, it is a habbit.“ - gerade in der Mittelohrchirurgie scheint derzeit das Erreichen eines höheren Qualitätsniveaus unweigerlich mit einer routinemäBigen Erfassung (Gewohnheit) und Beschreibung von Symptomen, Therapiemodalitäten und Bewertungsparametern einher zu gehen. Die mannigfaltigen Einflussfaktoren und Ausprägungen in Befund und Therapie machen langjährige und zentrumsübergreifende Datenkollektionen erforderlich, um ausreichend große Populationen für aussagekräftige Studien zu generieren. Dazu bedarf es zweifelsohne dem Bekenntnis der Fachwelt, vorhandene Bewertungssysteme zu verwenden, die Daten prospektiv in der Routine zu erfassen und zu pflegen. Diese Forderung mag, angesichts der aktuellen Arbeitsbelastung und empfundenen zunehmenden Arbeitsverdichtung in allen Bereichen, vermessen klingen, da sie ein „(noch) Mehr“ an Dokumentation bedeutet. Anderseits können nur so die Vorteile der digitalen Datenverarbeitung vollends genutzt werden. Die Implementierung der geeigneten Klassifikations- und Dokumentationssysteme in die tägliche Routine stellt somit die eigentliche Herausforderung dar, der wir uns stellen müssen. 


\section{Interessenkonflikt}

Der Autor gibt an, dass kein Interessenkonflikt besteht.

\section{Literatur}

[1] Kasperk R, Schumpelick V. Ergebnisqualität in der onkologischen Chirurgie. Chir 2002; 73: 545-549

[2] Donabedian A. The definition of quality: a conceptual exploration. In: Explorations in quality assessment and monitoring: The definition of quality and approaches to its assessment. Michigan: Ann Arbor: Health Administration Press; 1980: 3-31

[3] Tan HE, Santa Maria PL, Eikelboom RH, Anandacoomaraswamy KS, Atlas MD. Type I Tympanoplasty Meta-Analysis: A Single Variable Analysis. Otol Neurotol 2016; 37: 838-846

[4] Maxwell RJ. Quality assessment in health. BM] 1984; 288: 1470-1472

[5] Kestle JRW. Clinical Trials. World J Surg 1999; 23: 1205-1209

[6] Jalali MM, Motasaddi M, Kouhi A, Dabiri S, Soleimani R. Comparison of cartilage with temporalis fascia tympanoplasty: A meta-analysis of comparative studies: Cartilage Versus Fascia Tympanoplasty. The Laryngoscope 2017; 127: 2139-2148

[7] Mills R, Thiel G, Mills N. Results of myringoplasty operations in active and inactive ears in adults: Myringoplasty in Active and Inactive Ears. The Laryngoscope 2013; 123: 2245-2249

[8] Andersen SAW, Aabenhus K, Glad H, Sørensen MS. Graft take-rates after tympanoplasty: results from a prospective ear surgery database. Otol Neurotol 2014; 35: e292-e297

[9] Kirsten L, Morgenstern J, Erkkilä MT, Schindler M, Golde J, Walther ], Kemper M, Stoppe T, Bornitz M, Neudert M, Zahnert T, Koch E. Functional and morphological imaging of the human tympanic membrane with endoscopic optical coherence tomography. Curr Dir Biomed Eng 2017; 3: 99-101

[10] Kirsten L, Schindler M, Morgenstern J, Erkkilä MT, Golde J, Walther J, Rottmann P, Kemper M, Bornitz M, Neudert M, Zahnert T, Koch E. Endoscopic optical coherence tomography with wide field-of-view for the morphological and functional assessment of the human tympanic membrane. | Biomed Opt 2018; 24: 1

[11] Schindler M, Kirsten L, Morgenstern J, Golde J, Erkkilä M, Walther J, Kemper M, Bornitz M, Neudert M, Zahnert T, Koch E. Imaging of the human tympanic membrane by endoscopic optical coherence tomography. Curr Dir Biomed Eng 2018; 4: 305-308

[12] Hüttenbrink K-B. Die Funktion der Gehörknöchelchenkette und der Muskeln des Mittelohres. Laryngo-Rhino-Otol 1995

[13] Hüttenbrink K-B. Zur Rekonstruktion des Schallleitungsapparates unter biomechanischen Gesichtspunkten. Laryngo-Rhino-Otol 2000; 79: $23-51$

[14] Zahnert T. Laser in der Ohrforschung. Laryngo-Rhino-Otol 2003; 82 (Suppl 1): 157-180

[15] Zahnert T. Hearing disorder. Surgical management. Laryngo-RhinoOtol 2005; 84 (Suppl 1): S37-S50

[16] Beutner D, Hüttenbrink KB. Passive and active middle ear implants. Laryngorhinootologie 2009; 88 (Suppl 1): S32-S47

[17] Geyer G. Implants in middle ear surgery. Eur Arch Otorhinolaryngol Suppl 1992; 1: 185-221

[18] Ph Dost. Biomaterials in reconstructive middle ear surgery. Laryngo-Rhino-Otol 2000; 79: 53-72

[19] Hildmann H. Grundzüge einer differenzierten Cholesteatom-Chirurgie. Laryngo-Rhino-Otol 2000; 79: 73-94

[20] Schwager K. Reconstruction of the middle ear in abnormalities. Laryngorhinootologie 2007; 86 (Suppl 1): S141-S155
[21] Neudert M, Zahnert T, Lasurashvili N, Bornitz M, Lavcheva Z, Offergeld C. Partial ossicular reconstruction: comparison of three different prostheses in clinical and experimental studies. Otol Neurotol 2009; 30: 332-338

[22] Yu H, He Y, Ni Y, Wang Y, Lu N, Li H. PORP vs. TORP: a meta-analysis. Eur Arch Otorhinolaryngol 2013; 270: 3005-3017

[23] Zhang L-C, Zhang T-Y, Dai P, Luo J. Titanium versus non-titanium prostheses in ossiculoplasty: A meta-analysis. Acta Otolaryngol (Stockh) 2011; 131: 708-715

[24] Truy E, Naiman A, Pavillon C, Abedipour D, Lina-Granade G, Rabilloud M. Hydroxyapatite Versus Titanium Ossiculoplasty. Otol Neurotol 2007; 28: 492-498

[25] Zenner HP, Stegmaier A, Lehner R, Baumann I, Zimmermann R. Open Tübingen titanium prostheses for ossiculoplasty: a prospective clinical trial. Otol Neurotol 2001; 22: 582-589

[26] Neff BA, Rizer FM, Schuring AG, Lippy WH. Tympano-Ossiculoplasty Utilizing the Spiggle and Theis Titanium Total Ossicular Replacement Prosthesis. The Laryngoscope 2003; 113: 1525-1529

[27] Gardner EK, Jackson CG, Kaylie DM. Results with titanium ossicular reconstruction prostheses. Laryngoscope 2004; 114: 65-70

[28] Redaelli de Zinis LO. Titanium vs hydroxyapatite ossiculoplasty in canal wall down mastoidectomy. Arch Otolaryngol Head Neck Surg 2008; 134: 1283-1287

[29] Alaani A, Raut VV. Kurz titanium prosthesis ossiculoplasty - Followup statistical analysis of factors affecting one year hearing results. Auris Nasus Larynx 2010; 37: 150-154

[30] Plester D. The „old radical“ surgery. History and development of surgery of the mastoid. Laryngol Rhinol Otol (Stuttg) 1985; 64: 228-232

[31] Hildmann $\mathrm{H}$. The mastoid cavity and auditory canal meatoplasty. Relations between corresponding indications. Laryngol Rhinol Otol (Stuttg) 1986; 65: 684-687

[32] Stark T, Gurr A, Sudhoff H. Prinzipien der sanierenden Cholesteatomchirurgie. HNO 2011; 59: 393-400

[33] Mosher HP. A method of filling the excavated mastoid with a flap from the back of the auricle 1911; $21: 1158-1163$

[34] Palva T. Operative Technique In Mastoid Obliteration. Acta Otolaryngol (Stockh) 1973; 75: 289-290

[35] Dornhoffer JL, Smith J, Richter G, Boeckmann J. Impact on Quality of Life After Mastoid Obliteration. The Laryngoscope 2008; 118: $1427-1432$

[36] Kurien G, Greeff K, Gomaa N, Ho A. Mastoidectomy and mastoid obliteration with autologous bone graft: a quality of life study. J Otolaryngol - Head Neck Surg 2013; $42: 49$

[37] Lailach S, Kemper M, Lasurashvili N, Beleites T, Zahnert T, Neudert M. Health-related quality of life measurement after cholesteatoma surgery: comparison of three different surgical techniques. Eur Arch Otorhinolaryngol 2015; 272: 3177-3185

[38] Beutner D, Helmstaedter V, Stumpf R, Beleites T, Zahnert T, Luers JC, Huttenbrink K-B. Impact of Partial Mastoid Obliteration on Caloric Vestibular Function in Canal Wall Down Mastoidectomy. Otol Neurotol 2010; 31: 1399-1403

[39] Evans RA, Day GA, Browning GG. Open-cavity mastoid surgery: its effect on the acoustics of the external ear canal. Clin Otolaryngol Allied Sci 1989; 14: 317-321

[40] Hartwein J. Untersuchungen zur Akustik der offenen Mastoidhöhle (sog. „Radikalhöhle“) und deren Beeinflußbarkeit durch chirurgische Maßnahmen. Teil I: Physikalische Grundlagen, experimentelle Untersuchungen. Laryng Rhinol Otol 1992; 71: 401-406

[41] Jang $\mathrm{CH}$. Changes in external ear resonance after mastoidectomy: open cavity mastoid versus obliterated mastoid cavity. Clin Otolaryngol 2002; 27: 509-511 
[42] Shelton C, Sheehy JL. Tympanoplasty: review of 400 staged cases. The Laryngoscope 1990; 100: 679-681

[43] Whittemore KRJ, Merchant SN, Rosowski JJ. Acoustic mechanisms: Canal wall-up versus canal wall-down mastoidectomy. Otolaryngol Head Neck Surg 1998; 118: 751-761

[44] Gaihede M, Dirckx JJ], Jacobsen H, Aernouts J. Middle Ear Pressure RegulationVComplementary Active Actions of the Mastoid and the Eustachian Tube 2010; 31: 9

[45] Dircks J], Marcusohn Y, Gaihede M. Quasi-static Pressures in the Middle Ear Cleft. InSpringer Handbook of Auditory Research; 2013: 99-133

[46] Csakanyi Z, Katona G, Konya D, Mohos F, Sziklai I. Middle Ear Gas Pressure. Regulation: The Relevance of Mastoid Obliteration 2014; 35: 10

[47] van Dinther J, Vercruysse J-P, Camp S, De Foer B, Casselman J, Somers T, Zarowski A. Cremers CWRJ, Offeciers E. The Bony Obliteration Tympanoplasty in Pediatric Cholesteatoma. Long-term Safety and Hygienic Results. Otol Neurotol 2015; 36: 1504-1509

[48] Kang M-K, Ahn J-K, Gu T-W, Han C-S. Epitympanoplasty with mastoid obliteration technique: A long-term study of results. Otolaryngol Neck Surg 2009; 140: 687-691

[49] Lee WS, Choi JY, Song MH, Son EJ, Jung SH, Kim SH.. Mastoid and Epitympanic Obliteration in Canal Wall Up Mastoidectomy for Prevention of Retraction Pocket. Otol Neurotol 2005; 26: 1107-1111

[50] Black B. Mastoidectomy elimination. The Laryngoscope 1995; 105: $1-1$

[51] Schimanski GSE. Mastoid Cavity Obliteration with Bioactive Glass Granules. In: Lalwani AKPfister MHF, Hrsg. Recent advances in otolaryngology head \& neck surgery. Philadelphia: Jaypee Brothers Medical P; 2016; 5: 249-281

[52] Del Canizo SC. Radical surgery of the ear with free fat graft. Rev Clin Esp 1949; 34: 403

[53] Ringenberg JC, Fornatto E]. The Fat Graft in Middle Ear Surgery. Otolaryngol-Head Neck Surg 1962; 76: 407-413

[54] Moffat DA, Gray RF, Irving RM. Mastoid obliteration using bone pâté. Clin Otolaryngol Allied Sci 1994; 19: 149-157

[55] Gopalakrishnan S, Chadha SK, Gopalan G, Ravi D. Role of mastoid obliteration in patients with persistent cavity problems following modified radical mastoidectomy. J Laryngol Otol 2001; 115

[56] Silvola J, Palva T. Pediatric one-stage cholesteatoma surgery: long term results. Int J Pediatr Otorhinolaryngol 1999; 49: S87-S90

[57] Kirsch $\mathrm{H}$. Temporalis muscle grafts in the radical mastoid operation. J Laryng Otol 1928; 321-324

[58] Palva T. Reconstruction of ear canal in surgery for chronic ear. Arch Otolaryngol 1962; 75: 329-334

[59] Palva T, Palva A, Kärjä J. Musculoperiosteal flap in cavity obliteration: histopathological study seven years postoperatively. Arch Otolaryngol 1972; 172-177

[60] Ramsey M], Merchant SN, McKenna M]. Postauricular PeriostealPericranial Flap for Mastoid Obliteration and Canal Wall Down Tympanomastoidectomy. Otol Neurotol 2004; 25: 873-878

[61] Uçar C. Canal wall reconstruction and mastoid obliteration with composite multi-fractured osteoperiosteal flap. Eur Arch Otorhinolaryngol 2006; 263: 1082-1086

[62] Singh V, Atlas M. Obliteration of the Persistently Discharging Mastoid Cavity using the Middle Temporal Artery Flap. Otolaryngol Neck Surg 2007; 137: 433-438

[63] Yung M, Smith P. Mid-temporal pericranial and inferiorly based periosteal flaps in mastoid obliteration. Otolaryngol Neck Surg 2007; 137: 906-912

[64] Shiller A. „Mastoid osteoplasty“ using autologous cancellous bone. Arch Otolaryngol 1962; 75: 647-668
[65] Yung MW. The use of hydroxyapatite granules in mastoid obliteration. Clin Otolaryngol Allied Sci 1996; 21: 480-448

[66] Beutner D, Stumpf R, Zahnert T, Hüttenbrink K-B. Long-Term Results following Mastoid Obliteration in Canal Wall Down Tympanomastoidectomy. Laryngo-Rhino-Otol 2007; 86: 861-866

[67] Shea CM, Gardner G. Mastoid Obliteration Using Homograft Bone. Preliminary Report. Arch Otolaryngol 1970; 92: 358-365

[68] Shea MC, Gardner G, Simpson ME. Mastoid obliteration using homogenous bone chips and autogenous bone paste. Trans-Am Acad Ophthalmol Otolaryngol Am Acad Ophthalmol Otolaryngol 1972; 76: 160

[69] Solomons NB, Robinson JM. Obliteration of mastoid cavities using bone pâté. J Laryngol Otol 1988; 102: 783-784

[70] Shinkawa A, Sakai M, Tamura Y, Takahashi H, Ishida K. Canal-down tympanoplasty; one-stage tympanoplasty with mastoid obliteration, for non-cholesteatomatous chronic otitis media associated with osteitis. Tokai J Exp Clin Med 1998; 23: 19-23

[71] Roberson JB, Mason TP, Stidham KR. Mastoid obliteration: autogenous cranial bone pate reconstruction. Otol Neurotol 2003; 24: $132-140$

[72] Takahashi H, Iwanaga T, Kaieda S, Fukuda T, Kumagami H, Takasaki K, Hasebe S, Funabiki K. Mastoid obliteration combined with soft-wall reconstruction of posterior ear canal. Eur Arch Otorhinolaryngol 2007; 264: 867-871

[73] Vercruysse J-P, De Foer B, Somers T, Casselman JW, Offeciers E. Mastoid and Epitympanic Bony Obliteration in Pediatric Cholesteatoma. Otol Neurotol 2008; 29: 953-960

[74] Kuo C-Y, Huang B-R, Chen H-C, Shih C-P, Chang W-K, Tsai Y-L, Lin Y-Y, Tsai W-C, Wang C-H. Surgical Results of Retrograde Mastoidectomy with Primary Reconstruction of the Ear Canal and Mastoid Cavity. BioMed Res Int 2015; 2015: 1-12

[75] Levinson RM. Cartilage-perichondrial composite graft tympanoplasty in the treatment of posterior marginal and attic retraction pockets. Laryngoscope 1987; 97: 1069-1074

[76] Brask T. Obliteration of the mastoid cavities with crushed homograft cartilage in patients with cholesteatoma. Cholesteatoma and Mastoid Surgery. Kugler Ghedini 1989; 931-933

[77] Dornhoffer JL. Surgical Modification of the Difficult Mastoid Cavity. Otolaryngol Neck Surg 1999; 120: 361-367

[78] Kuo C-L, Lien C-F, Shiao A-S. Mastoid Obliteration for Pediatric Suppurative Cholesteatoma: Long-Term Safety and Sustained Effectiveness after 30 Years' Experience with Cartilage Obliteration. Audiol Neurotol 2014; 19: 358-369

[79] Kunert-Keil C, Kluge A, Kemper M, Zahnert T, Neudert M. Assessment of the osteogenic potential of human autologous bone dust - a cell and molecular biology pilot study. Barcelona 2017

[80] Kluge A, Neudert M, Kunert-Keil C, Lailach S, Zahnert T, Kemper M. The Obliteration of Noncritical Size Bone Defects With Bone Dust or Bone Replacement Material (Bioactive Glass S53P4). Otol Neurotol 2019; 40: e415-e423

[81] Mahendran S, Yung MW. Mastoid obliteration with hydroxyapatite cement: the Ipswich experience. Otol Neurotol Off Publ Am Otol Soc Am Neurotol Soc Eur Acad. Otol Neurotol 2004; 25: 19-21

[82] Park I-Y, Shimizu Y, O'Connor KN, Puria S, Cho J-H. Comparisons of electromagnetic and piezoelectric floating-mass transducers in human cadaveric temporal bones. Hear Res 2011; 272: 187-192

[83] Punke C, Zehlicke T, Boltze C, Pau HW. Experimental Studies on a New Highly Porous Hydroxyapatite Matrix for Obliterating Open Mastoid Cavities. Otol Neurotol 2008; 29: 807-811

[84] Lee H-B, Lim H], Cho M, Yang S-M, Park K, Park HY, Choung Y-H. Clinical Significance of $\beta$-Tricalcium Phosphate and Polyphosphate for Mastoid Cavity Obliteration during Middle Ear Surgery: Human and Animal Study. Clin Exp Otorhinolaryngol 2013; 6: 127 
[85] Yung M, Bennett A. Use of mastoid obliteration techniques in cholesteatoma. Curr Opin Otolaryngol Head Neck Surg 2013; 21: 455-460

[86] Walker PC, Mowry SE, Hansen MR, Gantz BJ. Long-term results of canal wall reconstruction tympanomastoidectomy. Otol Neurotol Off Publ Am Otol Soc Am Neurotol Soc Eur Acad. Otol Neurotol 2014; 35: e24-e30

[87] Estrem SA, Highfill G. Hydroxyapatite canal wall reconstruction/ mastoid obliteration. Otolaryngol - Head Neck Surg Off J Am Acad Otolaryngol-Head Neck Surg 1999; 120: 345-349

[88] Zoellner C, Buesing CM. How useful is tricalcium phosphate ceramic in middle ear surgery? Am J Otol 1986; 7: 289-293

[89] Reck R, Störkel S, Meyer A. Bioactive Glass-Ceramics in Middle Ear Surgery An 8-Year Review. Ann N Y Acad Sci 1988; 523: 100-106

[90] Reck R, Bernal Sprekelsen M. The effect of fibrin glue on the healing of hydroxyapatite ceramics. An animal experiment study. HNO 1989; 37: $112-116$

[91] Hartwein J, Hoermann K. A technique for the reconstruction of the posterior canal wall and mastoid obliteration in radical cavity surgery. Am J Otol 1990; 11: 169-173

[92] Yung MMW, Karia KR. Mastoid obliteration with hydroxyapatite-the value of high resolution $\mathrm{CT}$ scanning in detecting recurrent cholesteatoma. Clin Otolaryngol Allied Sci 1997; 22: 553-557

[93] Kupperman D, Tange RA. Ionomeric Cement in the Human Middle Ear Cavity: Long-Term Results of 23 Cases. The Laryngoscope 2001; 111: 306-309

[94] Dornhoffer J, Simmons O. Canal Wall Reconstruction with Mimix Hydroxyapatite Cement: Results in an Animal Model and Case Study. The Laryngoscope 2003; 113: 2123-2128

[95] D’Arc MB, Daculsi G, Emam N. Biphasic ceramics and fibrin sealant for bone reconstruction in ear surgery. Ann Otol Rhinol Laryngol 2004; 113: $711-720$

[96] Minoda R, Hayashida M, Masuda M, Yumoto E. Preliminary Experience With A-Tricalcium Phosphate for Use in Mastoid Cavity Obliteration After Mastoidectomy 2007; 28: 4

[97] Clark AE, Pantano CG, Hench LL. Auger Spectroscopic Analysis of Bioglass Corrosion Films. J Am Ceram Soc 1976; 59: 37-39

[98] Meuser W. Permanent obliteration of old radical mastoid cavities combined with tympanoplasty. J Laryngol Otol 1984; 98: 31-35

[99] Cho SW, Cho Y-B, Cho H-H. Mastoid Obliteration with Silicone Blocks after Canal Wall Down Mastoidectomy. Clin Exp Otorhinolaryngol 2012; 5: 23

[100] Jang CH, Cho YB, Bae CS. Evaluation of Bioactive Glass for Mastoid Obliteration: A Guinea Pig Model. In Vivo 2007; 5

[101] Stoor P, Pulkkinen J, Grénman R. Bioactive Glass S53P4 in the Filling of Cavities in the Mastoid Cell Area in Surgery for Chronic Otitis Media. Ann Otol Rhinol Laryngol 2010; 119: 377-382

[102] Sarin J, Grénman R, Aitasalo K, Pulkkinen J. Bioactive Glass S53P4 in Mastoid Obliteration Surgery for Chronic Otitis Media and Cerebrospinal Fluid Leakage. Ann Otol Rhinol Laryngol 2012; 121: 563-569

[103] Silvola JT. Mastoidectomy Cavity Obliteration with Bioactive. Glass: A Pilot Study. Otolaryngol Neck Surg 2012; 147: 119-126

[104] Harun A, Clark J, Semenov YR, Francis HW. The Role of Obliteration in the Achievement of a Dry Mastoid Bowl. Otol Neurotol 2015; 36: 1510-1517

[105] Clark MPA, Bottrill I. SerenoCemTM -glass ionomeric granules: a 3-year follow-up assessment of their effectiveness in mastoid obliteration. Clin Otolaryngol 2007; 32: 287-290

[106] Dornhoffer JL, Smith J, Richter G, Boeckmann J. Impact on Quality of Life After Mastoid Obliteration. The Laryngoscope 2008; 118: $1427-1432$
[107] Bernardeschi D, Russo FY, Nguyen Y, Canu G, Mosnier I, De Seta D, Ferrary E, Sterkers O. Management of epi- and mesotympanic cholesteatomas by one-stage trans-canal atticotomy in adults. Eur Arch Otorhinolaryngol 2016; 273: 2941-2946

[108] Uluyol S, Ugur O, Arslan IB, Yagiz O, Gumussoy M, Cukurova I. Effects of cavity reconstruction on morbidity and quality of life after canal wall down tympanomastoidectomy. Braz J Otorhinolaryngol 2018; 84: 608-613

[109] Yung M, Merkus P, Philips ], Black B, Tono T, Linder T, Dornhoffer ], Incesulu A. International Otology Outcome Group and the International Consensus on the Categorization of Tympanomastoid. Surgery. J Int Adv Otol 2018; 14: 216-226

[110] Tomlin J, Chang D, McCutcheon B, Harris J. Surgical Technique and Recurrence in Cholesteatoma: A Meta-Analysis. Audiol Neurotol 2013; 18: 135-142

[111] Trinidade A, Skingsley A, Yung MW. Mastoid obliteration surgery for cholesteatoma in 183 adult ears - a 5-year prospective cohort study: Our Experience. Clin Otolaryngol 2015; 40: 721-726

[112] Kuo C-L, Shiao A-S, Liao W-H, Ho C-Y, Lien C-F. How long is long enough to follow up children after cholesteatoma surgery? A 29-year study. The Laryngoscope 2012; 122: 2568-2573

[113] Mishiro Y, Sakagami M, Kitahara T, Kondoh K, Okumura S. The Investigation of the Recurrence Rate of Cholesteatoma Using Kaplan-Meier Survival Analysis. Otol Neurotol 2008; 29: 803-806

[114] van der Toom HFE, van der Schroeff MP, Pauw RJ. Single-Stage Mastoid Obliteration in Cholesteatoma Surgery and Recurrent and Residual Disease Rates: A Systematic Review. JAMA Otolaryngol-. Head Neck Surg 2018; 144: 440-446

[115] Roux A, Bakhos D, Lescanne E, Cottier J-P, Robier A. Canal wall reconstruction in cholesteatoma surgeries: rate of residual. Eur Arch Otorhinolaryngol 2015; 272: 2791-2797

[116] Trinidade A, Skingsley A, Yung MW. Pediatric Cholesteatoma Surgery Using a Single-Staged Canal Wall Down Approach. Results of a 5-Year Longitudinal Study 2015; 36: 4

[117] De Vos C, Gersdorff M, Gérard J-M. Prognostic factors in ossiculoplasty. Otol Neurotol Off Publ Am Otol Soc Am Neurotol Soc Eur Acad. Otol Neurotol 2007; 28: 61-67

[118] Bhattacharyya N. Outcomes Research in Otology. ORL 2004; 66 : 214-220

[119] Linstrom C, Silverman C, Rosen A, Meiteles L. Bone conduction impairment in chronic ear disease. Ann Otol Rhinol Laryngol 2001; 110: 437-441

[120] Tuz M, DoÄ̈̈ru H, Uygur K, Gedikli O. Improvement in bone conduction threshold after tympanoplasty. Otolaryngol Head Neck Surg 2000; 123: 775-778

[121] Vartiainen E, Seppa J. Results of bone conduction following surgery for chronic ear disease. Eur Arch Otorhinolaryngol 1997; 254: 384-386

[122] Black B. Reporting results in ossiculoplasty. Otol Neurotol 2003; 24: 534-542

[123] AAO-HNS Committee on Hearing and Equilibrium guidelines for the evaluation of results of treatment of conductive hearing loss. Otolaryngol Head Neck Surg 1995; 113: 186-187

[124] Goldenberg RA, Berliner KI. Reporting operative hearing results: does choice of outcome measure make a difference? Otol Neurotol 1995; 16: $128-135$

[125] Plontke SK, Girndt M, Meisner C, Probst R, Oerlecke I, Richter M, Steighardt J, Dreier G, Weber A, Baumann I, PlößI S, Löhler J, Laszig R, Werner JA, Rahne T. Multizentrische Studie zur Hörsturztherapie Planung und Konzeption. HNO 2016; 64: 227-236

[126] Hornsby BWY. The Speech Intelligibility Index: What is it and what's it good for? Hear | 2004; 57: 6 
[127] Martin FN, Champlin CA, Chambers JA. Seventh survey of audiometric practices in the United States. J Am Acad Audiol 1998; 9: 95-104

[128] Müller J, Plontke SK, Rahne T. Sprachaudiometrische Zielparameter in klinischen Studien zur Hörverbesserung. HNO 2017; 65: 211-218

[129] Kamm CA, Morgan DE, Dirks DD. Accuracy of adaptive procedure estimates of PF-max level. J Speech Hear Disord 1983; 48: 202-209

[130] Gurgel RK, Jackler RK, Dobie RA, Popelka GR. A new standardized format for reporting hearing outcome in clinical trials. Otolaryngol Head Neck Surg 2012; 147: 803-807

[131] Lailach S, Baumann I, Zahnert T, Neudert M. Aktueller Stand der Lebensqualitätsmessung bei Patienten mit chronischer Otitis media und Schallleitungsschwerhörigkeit. HNO 2018; 66: 578-589

[132] Baumann I, Kurpiers B, Plinkert P, Praetorius M. Development and validation of the Chronic Otitis Media Outcome Test 15 (COMOT-15). Measurement of health-related quality of life in patients with chronic otitis media. HNO 2009; 57: 889-895

[133] Neudert M, Zahnert T. Tympanoplastik - Neues und neu Beleuchtetes. Laryngo-Rhino-Otol 2017; 91: S66-S83

[134] Aiello CP, Lima II de Ferrari DV. Validity and reliability of the hearing handicap inventory for adults. Braz J Otorhinolaryngol 2011; 77 : 432-438

[135] Baba S, Ikezono T, Pawankar R, Yagi T. Congenital Malformations of the Middle Ear with an Intact External Ear: A Review of 38 Cases. ORL 2004; 66: 74-79

[136] Nadol JB, Staecker H, Gliklich RE. Outcomes assessment for chronic otitis media: the Chronic Ear Survey. The Laryngoscope 2000; 110: 32-35

[137] Lailach S, Schenke T, Baumann I, Walter H, Praetorius M, Beleites T, Zahnert T, Neudert M. Development and validation of the Stapesplasty Outcome Test 25 (SPOT-25). HNO 2017

[138] Baumann I, Gerendas B, Plinkert PK, Praetorius M. General and disease-specific quality of life in patients with chronic suppurative otitis media - a prospective study. Health Qual Life Outcomes 2011; 9: 48

[139] Nadol JB, Staecker H, Gliklich RE. Outcomes Assessment for Chronic Otitis Media: The Chronic Ear Survey: Outcomes Assessment for Chronic Otitis Media: The Chronic Ear Survey. The Laryngoscope 2000; $110: 32-35$

[140] Robinson K, Gatehouse S, Browning GG. Measuring Patient Benefit from Otorhinolaryngological Surgery and Therapy. Ann Otol Rhinol Laryngol 1996; 105: 415-422

[141] Reetz T, Lailach S, Garthus-Niegel S, Neudert M, Zahnert T. Impact of depressiveness on disease-specific quality of life $(\mathrm{QoL})$ in patients with chronic otitis media. Estrel Congress Center Berlin. 2019, s-0039-1686478. Im Internet: http://www.thieme-connect.de/DOI/ DOI? doi:10.1055/s-0039-1686478

[142] Neudert M. Health related quality of life measures as outcome parameters in middle ear diseases. ENT Audiol News 2018; 26: 2-4

[143] Schwager K. Akute Komplikationen in der Mittelohrchirurgie: Teil 1: Probleme während der Tympanoplastik - was tun? HNO 2007; 55: 307-317

[144] Schick B, Dlugaiczyk J. Surgery of the ear and the lateral skull base: pitfalls and complications. GMS Curr Top Otorhinolaryngol. Head Neck Surg 2013; 12 Doc05

[145] Linder TE, Lin F. Felsenbeinchirurgie: Komplikationen und unerwünschte Operationsfolgen. HNO 2011; 59: 974-979

[146] Thomeczek C, Bock W, Ekkernkamp A, Everz D, Fischer D, Gerlach F, Gibis B, Gramsch E, Jonitz G, Klakow-Frank R, Oesingmann U, Schirmer H, Smentkowski U, Ziegler M, Ollenschläger G. Das Glossar Patientensicherheit. Ein Beitrag zur Definitionsbestimmung und zum Verständnis der Thematik „Patientensicherheit“ und „Fehler in der Medizin“. Gesundheitswesen 2004; 833-840
[147] Sokol DK, Wilson J. What is a Surgical Complication? World J Surg 2008; 32: 942-944

[148] Dindo D, Clavien P-A. What Is a Surgical Complication? World J Surg 2008; 32: 939-941

[149] Lailach S, Enders ], Zahnert T, Neudert M. Complications after middle ear reconstruction surgery - Is a preoperative or perioperative risk stratification intended? 2019; 98: S140

[150] Kazikdas KC, Onal K, Yildirim N. Sensorineural hearing loss after ossicular manipulation and drill-generated acoustic trauma in type I tympanoplasty with and without mastoidectomy: A series of 51 cases. Ear Nose Throat J 2015. Im Internet: http://www.entjournal. com/article/sensorineural-hearing-loss-after-ossicular-manipulationand-drill-generated-acoustic-trauma-

[151] Wullstein $\mathrm{H}$. Theory and practice of tympanoplasty. Laryngoscope 1956; 66: 1076-1093

[152] Bellucci R. Basic considerations for success in tympanoplasty. Arch Otolaryngol 1969; 90: 732-741

[153] Black B. Ossiculoplasty prognosis: the spite method of assessment. Am J Otol 1992; 13: 544-551

[154] Austin D. Types and indications of staging. Arch Otolaryngol 1969; 89: 235-242

[155] Kartush JM. Ossicular chain reconstruction. Capitulum to Malleus. Otolaryngol Clin North Am 1994; 27: 689-715

[156] Becvarovski Z, Kartush JM. Smoking and tympanoplasty: implications for prognosis and the Middle Ear Risk Index (MERI). Laryngoscope 2001; 111: 1806-1811

[157] Dornhoffer JL, Gardner E. Prognostic factors in ossiculoplasty: a statistical staging system. Otol Neurotol 2001; 22: 299-304

[158] Tos M. Cartilage Tympanoplasty Methods: Proposal of a Classification. Otolaryngol Neck Surg 2008; 139: 747-758

[159] Cohen MS, Basonbul RA, Barber SR, Kozin ED, Rivas AC, Lee D]. Development and validation of an endoscopic ear surgery classification system. The Laryngoscope 2018; 128: 967-970

[160] Rutkowska J, Ozgirgin N, Olszewska E. Cholesteatoma Definition and Classification: A Literature Review. J Int Adv Otol 2017; 13: 266-271

[161] Yung M, Tono T, Olszewska E, Yamamoto Y, Sudhoff H, Sakagami M, Mulder J, Kojima H, Incesulu A, Trabalzini F, Ozgirgin N. EAONO/JOS Joint Consensus Statements on the Definitions, Classification and Staging of Middle Ear Cholesteatoma. J Int Adv Otol 2017; 13: 1-8

[162] Lailach S, Zahnert T, Neudert M. Data and Reporting Quality in Tympanoplasty and Ossiculoplasty Studies. Otolaryngol - Head Neck Surg Off J Am Acad Otolaryngol-Head Neck Surg 2017; 157: 281-288

[163] Gardner G, Robertson JH. Hearing preservation in unilateral acoustic neuroma surgery. Ann Otol Rhinol Laryngol 1988; 97: 55-66

[164] Jackler RK. Comparability in reporting outcomes: A scientific imperative. Am J Otol 1996; 17: 811-812

[165] Wullstein $\mathrm{H}$. The restoration of the function of the middle ear, in chronic otitis media. Ann Otol Rhinol Laryngol 1956; 65: 1021-1041

[166] Wullstein $\mathrm{H}$. Theory and practice of tympanoplasty. Laryngoscope 1956; 66: 1076-1093

[167] Bellucci R. Basic considerations for success in tympanoplasty. Arch Otolaryngol 1969; 90: 732-741

[168] Bellucci R. Dual classification of tympanoplasty. Laryngoscope 1973; 83: $1754-1758$

[169] Austin DF. Types and indications of staging. Arch Otolaryngol Chic III 19601969 89: 235-242

[170] Lailach S, Zahnert T, Neudert M. Data and Reporting Quality in Tympanoplasty and Ossiculoplasty Studies. Otolaryngol - Head Neck Surg Off J Am Acad Otolaryngol-Head Neck Surg 2017; 157: 281-288 
[171] Schön F. Müller. J. Der Würzburger Ohrbogen: Ein System zur Dokumentation von Operationen und Nachsorgeuntersuchungen in der HNO-Heilkunde. Laryngo-Rhino-Otol 2002; 81: 171-177

[172] Wullstein HL. Operationen zur Verbesserung des Gehörs. Stuttgart: G. Thieme; 1968

[173] Wullstein HL, Bandtlow O, Kreutle OJ, Schmitt HG. Erfahrungen Bei Der Statistischen Auswertung Gehörverbessernder Operationen Mit Dem Ibm Lochkartenver-Fahren. Acta Otolaryngol (Stockh) 1962; 54 : 56-74

[174] Schmitt HG. Die elektronische Datenverarbeitung in der klinischen Medizin am Beispiel der Operationen zur Verbesserung des Gehörs. 1967

[175] Yung M, Smith P, Hausler R, Martin C, Offeciers E, Pytel J, Skladzien ], Somers T, Ven de Heyning P. International Common Otology Database: Taste Disturbance After Stapes Surgery. Otol Neurotol 2008; 29: 661-665

[176] Yung M, Gjuric M, Haeusler R, Van de Heyning PH, Martin C, Swan IRC, Tange RA, Ba Huy PT. An International Otology Database: Otol Neurotol 2005; 26: 1087-1092

[177] Kim H-J. A standardized database management of middle ear surgery in Korea. Acta Otolaryngol (Stockh) 2007; 127: 54-60

[178] Moriyama H, Yamamoto E, Yuasa E. Classification and nomenclature of ossicular reconstruction. Otol Jpn 2001; 11: 64-68

[179] Austin DF. Ossicular reconstruction. Arch Otolaryngol 1971; 94 : 525-535

[180] Farrior ], Nichols S. Long-term results using ossicular grafts. Am J Otol 1996; 17: 386-392

[181] Maeng JW, Kim H-J. Effects of Middle Ear Lesions on Pre and Postoperative Hearing Outcomes in Patients with Chronic Otitis Media. Korean J Audiol 2012; 16: 18

[182] Rombout J, Moorman PW, Holm AF, Pauw KH. The methodical collection of ear surgery data as a basis for quality control. Eur Arch Otorhinolaryngol 2002; 259: 184-192

[183] Vincent R, Sperling NM, Oates ], Jindal M. Surgical Findings and Long-Term Hearing Results in 3,050 Stapedotomies for Primary Otosclerosis: A Prospective Study with the Otology-Neurotology. Database 2006; 27: 23

[184] Berglund M, Florentzson R, Fransson M, Hultcrantz M, Eriksson PO, Englund E, Westman E. Myringoplasty Outcomes From the Swedish National Quality Registry: Myringoplasty Outcomes in a Swedish Database. The Laryngoscope 2017; 127: 2389-2395

[185] Herisanu IT, Hoth S, Praetorius M. Das Heidelberger Cl-Datenbankmodul: Qualitätssicherung in der Cochleaimplantatversorgung. HNO 2016; 64: 891-896

[186] Rompaey VV, Yung M, Claes ], Hausler R, Martin C, Skladzien J, de Heyning PV. Prospective Effectiveness of Stapes Surgery for Otosclerosis in a Multicenter Audit Setting: Feasibility of the Common Otology Database as a Benchmark. Database. 2009; 30: 10
[187] Phillips JS, Yung MW, Nunney I. Myringoplasty outcomes in the UK. J Laryngol Otol 2015; 129: 860-864

[188] Van Rompaey V, Claes G, Potvin J, Wouters K, Van de Heyning P. Systematic Review of the Literature on Nitinol Prostheses in Surgery for Otosclerosis: Assessment of the Adequacy of Statistical Power. Otol Neurotol 2011; 32: 357-366

[189] Caremans ], Hamans E, Muylle L, Van de Heyning P, Van Rompaey V. Endoscopic versus transcranial procurement of allograft tympanoossicular systems: a prospective double-blind randomized controlled audit. Cell Tissue Bank 2016; 17: 199-204

[190] Kim H-J. A standardized database management of middle ear surgery in Korea. Acta Otolaryngol (Stockh) 2007; 127: 54-60

[191] Vincent R, Rovers M, Zingade N, Oates J, Sperling N, Devèze A, Grolman W. Revision Stapedotomy: Operative Findings and Hearing Results. A Prospective Study of 652 Cases From the Otology-Neurotology Database. Otol Neurotol 2010; 31: 875-882

[192] Vincent R, Bittermann AJN, Oates J, Sperling N, Grolman W. KTP Versus CO2 Laser Fiber Stapedotomy for Primary Otosclerosis: Results of a New Comparative Series With the Otology-Neurotology Database. 2012; 33: 6

[193] Vincent R, Grolman W, Oates ], Sperling N, Rovers M. A nonrandomized comparison of potassium titanyl phosphate and CO 2 laser fiber stapedotomy for primary otosclerosis with the otology-neurotology database. The Laryngoscope 2010; 120: 570-575

[194] Vincent R, Bittermann AJN, Wenzel G, Oates ], Sperling N, Lenarz T, Grolman W. Ossiculoplasty in missing malleus and stapes patients: experimental and preliminary clinical results with a new malleus replacement prosthesis with the otology-neurotology database. Otol Neurotol 2013; 34: 83-90

[195] Aabenhus K, Andersen SAW, Sørensen MS. Hearing Results After Tympanoplasty Are Stable Short-term: A Prospective Database Study. Otol Neurotol 2016; 37: 1335-1343

[196] Andersen SAW, Öhman MC, Sørensen MS. The stability of short-term hearing outcome after stapedotomy: a prospective database study. Acta Otolaryngol (Stockh) 2015; 135: 871-879

[197] Strömbäck K, Lundman L, Bjorsne A, Grendin J, Stjernquist-Desatnik A, Dahlin-Redfors Y. Stapes surgery in Sweden: evaluation of a national-based register. Eur Arch Otorhinolaryngol 2017; 274: 2421-2427

[198] Berglund M, Suneson P, Florentzson R, Fransson M, Hultcrantz M, Westman E, Eriksson PO. Tinnitus and taste disturbances reported after myringoplasty: Data from a national quality registry: Tinnitus and Taste After Myringoplasty. The Laryngoscope 2019; 129: 209-215

[199] Maile EJ, Tharu PB, Blanchford HLK, Edmiston R, Youngs R. Quality of life of Nepali patients with ear disease before and after corrective surgery. Trop Med Int Health 2015; 20: 1041-1047

[200] Hazenberg AJC, Hoppe FF, Dazert S, Minovi A. Lebensqualitätbewertung nach Stapesoperationen. HNO 2013; 61: 233-239 